\title{
Chapter 2 \\ Scattering of Soft Condensed Matter: \\ From Fundaments to Application
}

\author{
N. Stribeck
}

\begin{abstract}
Scattering data recorded in experiments on soft condensed matter are resulting in continuous scattering patterns which must be processed for structure analysis. The review presents scattering theory as a tool box with examples that show how to use it in practice. For some of the operations commented source code for computer programming is given. The focus is on the application to polymer and related materials.
\end{abstract}

\subsection{Introduction}

In the past decade experimental technique has been on a fast pace, whereas the development of data evolution methods is proceeding slowly. In fact, most of the progress of 30 years achieved in the field of method has not even been disseminated, and the skills required to transform methodical ideas into computer programs appear to be declining. Thus, it is not astonishing to find more and more publications based on the interpretation of untreated raw data, even if it would have been rewarding "to cut the raw diamond". Hence, there is good reason to try and lower the threshold both with respect to theory and with respect to the development of practical algorithms.

Scientists who are aware of this problem are frequently asking why there is no standard and powerful computer program for the evaluation of scattering data, similar to the more favorable situation found in protein crystallography. There are several answers to this question.

The first and grave answer is provided by the fundamental difference between diffraction and scattering, which has already been emphasized by Debye and Menke [1] 80 years ago: Even though both the diffraction pattern of crystal structure and

\footnotetext{
N. Stribeck

Institute of Technical and Macromolecular Chemistry, Bundesstr. 45, D-20146 Hamburg, Germany, Norbert.Stribeck@desy.de
} 
the scattering of soft matter provide the power spectrum $^{1}$ in reciprocal space of the respective structure, the structure of the crystal is mathematically described by a discrete ${ }^{2}$ and periodical function, whereas the nanostructure of soft matter is, in general, a continuous function with short range of order. While crystal structure is accurately determined by direct and straightforward analysis of a finite number of the peaks that make the pattern, soft matter nanostructure can only be analyzed with some significance if the complete pattern is taken into account. In the area of soft matter scattering there is not even a straightforward method of analysis - except for several kinds of back-transformations to real space. Moreover, if it is decided to stay in reciprocal space, an adequate model for the structure must be at hand to fit the scattering pattern.

The second answer is provided by the inherent heterogeneity of the problems to be analyzed. While the crystallographic experiment and its analysis are standardized, in materials science scientific creativity is blossoming in the area of experimental technique. Moreover, in this roaring progress even the few approved design standards are frequently disobeyed. As a consequence, the experiment aimed at by the analysis program is a running target.

The third answer is a blunt callback: If the scientific community itself is not willing to develop powerful tools for data analysis, who else would do so? Consequently, the aim of this chapter is a demonstration of geometry, theory, and related practical examples.

\subsection{Experiment Geometry and Reciprocal Space}

The most frequent experimental setup for X-ray scattering experiments of soft matter samples is sketched in Fig. 2.1. It is called the normal transmission setup. With

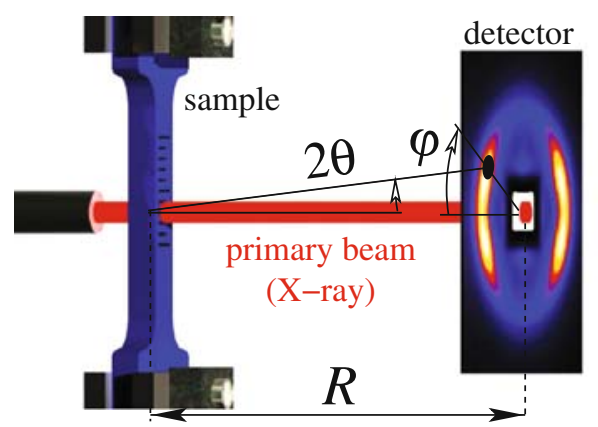

Fig. 2.1 Normal transmission setup of an X-ray scattering experiment. The intensity at a chosen point (circular mark) on the planar detector, $I(2 \theta, \varphi)$ is a function of the scattering angle $2 \theta$ and the azimuthal angle $\varphi . R$ is the sample-detector distance

\footnotetext{
${ }^{1}$ More exactly: the power spectral density.

${ }^{2}$ In the gap between the atoms there is a vacuum.
} 
respect to the X-ray primary beam, sample and detector are oriented at right angles (normal). The primary beam penetrates a relatively thin sample and the scattering pattern is recorded around the attenuated beam "in transmission". By means of a planar detector the scattering intensity $I(x, y)$ is recorded. The sample-detector distance, $R$, is the main parameter which controls the angular range in which the scattering is recorded. In fact, the area of X-ray scattering is subdivided into several subareas which are characterized by a typical range of scattering angles or distances $R$ (Table 2.1). As deduced from the sketch, every pair of scattering-related coordinates, $(2 \theta, \varphi)$, is readily addressed by pixel coordinates ${ }^{3}(x, y)$ on the detector

$$
\begin{aligned}
\tan 2 \theta & =\sqrt{x^{2}+y^{2}} / R \\
\tan \varphi & =y / x,
\end{aligned}
$$

with the scattering angle $2 \theta$, the azimuthal angle $\varphi$, and the sample-detector distance $R$.

As we are carrying out a scattering experiment, we are interested in the relation of the measured scattering intensity to the structure $\rho(\mathbf{r})$ inside the volume $V$ that is irradiated by the X-ray beam.

In the field of X-ray scattering, $\rho(\mathbf{r})$ is the electron density, because X-rays are interacting with the electrons of matter. Here $\mathbf{r}=\left(r_{1}, r_{2}, r_{3}\right)$ is the vector in real space. Only if $\rho(\mathbf{r})$ is varying inside the irradiated volume, X-rays are scattered off the primary beam. Materials entities which share the same electron density cannot be discriminated by means of X-ray scattering.

\section{Reciprocal Space}

A result of the kinematic scattering theory is the fact that the relation between the structure of matter and the scattered intensity is treated to the best advantage if the latter is regarded with respect to a so-called reciprocal space $\mathbf{s}=\left(s_{1}, s_{2}, s_{3}\right)$, i.e.,

$$
I(2 \theta, \varphi) \rightarrow I(\mathbf{s})
$$

Table 2.1 Subareas of X-ray scattering as a function of the sample-detector distance $R$ assuming a wavelength $\lambda \approx 0.15 \mathrm{~nm}$

\begin{tabular}{lll}
\hline Subarea & $R[\mathrm{~m}]$ & Focus \\
\hline WAXS & $0.05-0.2$ & Arrangement of chain segments \\
MAXS & $0.2-1$ & Liquid-crystalline structure \\
SAXS & $1-3$ & Nanostructure $3-50 \mathrm{~nm}$ \\
USAXS & $6-15$ & Nanostructure $15-2 \mu \mathrm{m}$ \\
\hline
\end{tabular}

\footnotetext{
${ }^{3}$ Here it is assumed that the center of the primary beam on the detector is at $x=y=0$.
} 


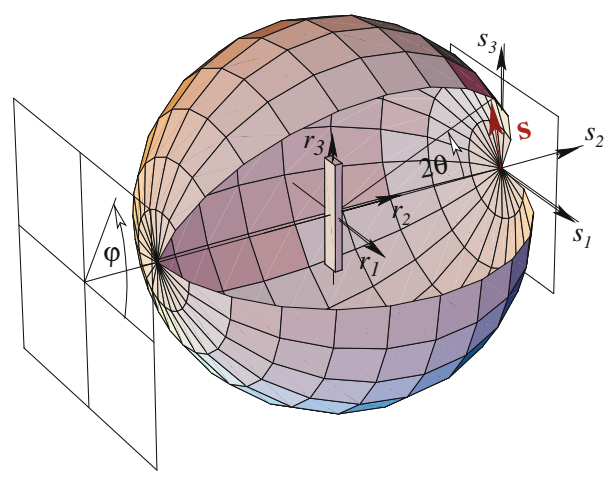

Fig. 2.2 Experiment geometry, Ewald sphere, and reciprocal space. The sample is mounted in the center of the sphere. Its structure is defined in the real-space coordinates, $\mathbf{r}=\left(r_{1}, r_{2}, r_{3}\right)$. The origin of reciprocal space, $\mathbf{s}=\left(s_{1}, s_{2}, s_{3}\right)$, is attached to the surface of the Ewald sphere. The orientation of its axes is strictly coupled to the orientation of the sample space $\mathbf{r}$. Each point on a plane detector (for clarity shifted to a position in front of the sphere) can be addressed by the two coordinates scattering angle, $2 \theta$, and azimuthal angle, $\varphi$. The mapping of these two coordinates to reciprocal space is mediated by means of the surface of the Ewald sphere

Figure 2.2 explains the two-step geometric relation ranging from the sample coordinate system in real space over the definition of reciprocal space finally to the scattering intensity measured on the detector's plane. A simple formal analysis of Eq. (2.1) shows that it maps a plane into reciprocal space. In fact, this mapping is nonlinear and the image of the infinite detector plane in reciprocal space is the surface of the sphere indicated in Fig. 2.2. It is called the Ewald sphere.

In the center of the Ewald sphere is the sample in its real-space coordinate system $\mathbf{r}=\left(r_{1}, r_{2}, r_{3}\right)$. Let the primary beam be propagating from left to right. Assuming an infinitesimal irradiated volume $V$ in the center of the sphere, scattering $I(2 \theta, \varphi)$ is observed. The origin of reciprocal space, $\mathbf{s}=\left(s_{1}, s_{2}, s_{3}\right)$, is attached to the point where the primary beam which has already penetrated the sample breaks through the Ewald sphere. The Cartesian axes of reciprocal space are strictly parallel to the axes $\left(r_{1}, r_{2}, r_{3}\right)$, the coordinate system of (physical) real space. ${ }^{4}$ Thus, by rotating the sample $\mathrm{e}^{5}$ in the beam we can probe a considerable fraction of reciprocal space, in which Ewald's sphere is the representation of the (infinite) detector plane.

\footnotetext{
${ }^{4}$ This isomorphism of real and reciprocal space in the area of scattering is a consequence of the definition of the basis vectors in real space by $r_{i} r_{j}=\delta_{i j}$, resulting in a basis made of ortho-normal unit vectors. From this definition (using Kronecker's symbol $\delta_{i j}$ ) it follows that the dual (i.e., mutually reciprocal) bases are mutually identical, with duality defined by

$$
r_{i} s_{j}=\delta_{i j}
$$

In crystallography the definition is different, as the corresponding basis vectors are usually defined by the edges of the crystal unit cell. Then the basic relations between crystal structure and the position of diffraction peaks are readily established from Eq. (2.2).

${ }^{5}$ Only the sample but not the detector is rotated.
} 
For a general structure to be studied, we will have to perform this sample rotation "about the two Euler angles" in order to collect the information that is required for a structure analysis. Fortunately, soft materials frequently show some symmetry of the scattering intensity $I(\mathbf{s})$, and every kind of symmetry is reducing the necessary effort.

Isotropy and the Normalization of Reciprocal Space

In the most simple case of isotropic scattering the scattering intensity

$$
I(\mathbf{s})=I(s)
$$

is only a function of the modulus of the s-vector, $s=\sqrt{s_{1}^{2}+s_{2}^{2}+s_{3}^{2}}$. On the plane detector the intensity is no longer a function of the azimuthal angle $\varphi$, and in the most convenient definition of reciprocal space, $s$ is related to the scattering angle by

$$
s=\frac{2}{\lambda} \sin \theta
$$

with $\lambda$ the wavelength of the X-radiation. A wavelength typically chosen for the study of soft matter is $\lambda \approx 0.15 \mathrm{~nm}^{-1}$. Quite popular is another definition of reciprocal space in terms of a vector $\mathbf{q}$, the magnitude of which is related to $s$ by

$$
q=2 \pi s
$$

In the case of isotropy all the intensity maxima on the plane detector are rings ("Debye-Scherrer rings"). Then the complete information on the scattering is in a radial cut (slice) extending outward from the center of the pattern. Nevertheless, it is good practice to take advantage of the complete recorded pattern by performing an azimuthal average of the scattering pattern. Thus, the noise in the extracted curve is reduced considerably as compared to the sliced curve. The operation can directly be performed by the free evaluation program FIT2D ([2], Chap. 11).

\section{Fiber Symmetry}

Materials with fiber symmetry

$$
I(\mathbf{s})=I\left(s_{12}, s_{3}\right)
$$

combined with an X-ray scattering setup equipped with a 2D detector are an excellent match for a study of structure evolution. The reason is the fact that (almost) the complete scattering information from reciprocal space can be recorded in a single exposure. In Eq. (2.6), $s_{12}=\sqrt{s_{1}^{2}+s_{2}^{2}}$ is the transverse coordinate in cylindrical coordinates, and $s_{3}$ is the longitudinal coordinate of reciprocal space "in 
fiber direction". Among other materials, fiber symmetry is shown by polymer fibers, elongated elastomers, and materials under uniaxial mechanical load.

In order to exploit fiber symmetry to its maximum content, the detector should record a representative plane of reciprocal space, e.g., $\left(s_{1}, s_{3}\right)$, as indicated in Fig. 2.2 on the right side by means of a rectangular frame. Unfortunately the detector is the Ewald sphere, and this sphere is bending off the $\left(s_{1}, s_{3}\right)$-plane. Small is the error close to the vicinity of the origin, i.e., for ultra-small (USAXS) and small (SAXS) scattering angles. This region is said to conform to the tangent plane approximation, because in this angular region the Ewald sphere is well approximated by its tangential $\left(s_{1}, s_{3}\right)$-plane.

For bigger scattering angles (MAXS and WAXS) the mapping of the detector on the $\left(s_{1}, s_{3}\right)$-plane results in blind areas on the meridian $\left(s_{3}\right.$-axis) of the pattern, whereas on the equator ( $s_{12}$-direction) the geometrical warping of the intensity is small. For MAXS and WAXS experiments it is often beneficial to tilt the fiber sample with respect to the primary beam. The tilt angle $\theta^{\prime}$ should be half the scattering angle $2 \theta^{\prime}$, at which interesting scattering is expected on the meridian. The warped mapping of a square detector grid on the $\left(s_{1}, s_{3}\right)$-plane is a geometrical exercise that is readily deduced from Fig. 2.2. Although its basics and the related Bernal chart are already documented in the textbook of Alexander [3], it is now frequently addressed by the term Fraser correction, referring to the first publication [4] of its application in digital data evaluation of fiber patterns. The result of such a mapping is shown in Fig. 2.3. Thus, by recording a series of images taken at different tilt angles of the fiber the blind area can be covered to a sufficient extent. Finally, the remnant blind areas may be covered from symmetry consideration or by means of $2 \mathrm{D}$ extrapolation procedures, e.g., the algorithm based on radial basis functions [5].

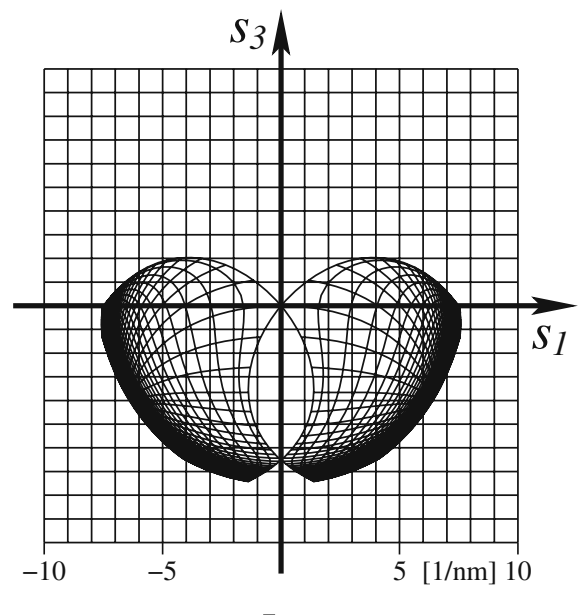

Fig. 2.3 WAXS, 2D detector, and fiber symmetry: unwarping of the detector surface to map it on the $\left(s_{1}, s_{3}\right)$-plane. The fiber sample is tilted by $\theta^{\prime}=40^{\circ}$ with respect to the primary beam. $R=8 \mathrm{~cm}, \lambda=0.154 \mathrm{~nm}$. The apparent warped grid has been a square grid on the detector (edge length: $2 \mathrm{~cm}$ ) 


\subsection{Materials Structure and Scattering Pattern}

In order to establish the relation between the structure of matter and the corresponding X-ray scattering pattern a two-step deduction is carried out (cf. textbooks, e.g., $[3,6-9])$.

In the first step the interaction of X-rays with a single electron (in some absorbing matter) is considered, elaborating all the deviations of the practical interaction from the paradigm of a photon that is interacting elastically with a pseudo-electron that, in return, is emitting a spherical wave. As a result, several corrections should be applied to the measured raw data before a quantitative analysis ${ }^{6}$ in order to make them satisfy the paradigm. These corrections comprise absorption correction, polarization correction, correction for Compton scattering, suppression of fluorescence, and multiple scattering [10]. In practice, absorption correction should be applied in general. Polarization correction is important for the WAXS, as is a subtraction of the Compton scattering background [3]. A test for multiple scattering [10] is important if porous materials are studied by SAXS. It is very important in the field of USAXS.

In the second step the arrangement of the electrons (i.e., the structure of matter) is introduced, and the Fourier relation of the structure to the scattered X-ray intensity $I(\mathbf{s})$ is established by the so-called kinematic scattering theory.

\section{The Fourier Transform}

According to the Fraunhofer approximation of kinematic scattering theory the real space and the reciprocal space are related to each other by an integral transform known by the name Fourier transform. It shall be indicated by the operator $\mathscr{F}()$. The $n$-dimensional $(\mathrm{nD})$ Fourier transform of $h(r)$ is defined by

$$
\mathscr{F}_{n}(h)(\mathbf{s}):=\int h(\mathbf{r}) \exp (2 \pi i \mathbf{r s}) d^{n} r,
$$

with $i$ the imaginary unit, and back-transformation simply yields

$$
\mathscr{F}_{-n}(H)(\mathbf{r}):=\int H(\mathbf{s}) \exp (-2 \pi i \mathbf{r s}) d^{n} s,
$$

with $H(\mathbf{s}):=\mathscr{F}_{n}(h)(\mathbf{s})$. In the field of scattering 1D, 2D, and 3D transforms are required. The kernel of the Fourier transform is called the harmonic function

$$
\exp (2 \pi i r s)=\cos (2 \pi r s)+i \sin (2 \pi r s)
$$

and the Fourier transform is said to perform an harmonic analysis.

In the q-system the pair of transformations

$$
\mathscr{F}_{n}(h)(\mathbf{q}):=\int h(\mathbf{r}) \exp (i \mathbf{r q}) d^{n} r
$$

\footnotetext{
${ }^{6}$ Quantitative analysis means that the shape of the intensity function is considered or entered in a numerical evaluation procedure. For a simple qualitative discussion (peak positions, strong intensity changes) corrections are negligible.
} 
and

$$
\mathscr{F}_{-n}(H)(\mathbf{r}):=\left(\frac{1}{2 \pi}\right)^{n} \int H(\mathbf{q}) \exp (-i \mathbf{r q}) d^{n} q
$$

is asymmetric with different pre-factors that must be tracked in calculus.

Structure and Scattering in a Nutshell.

The fundamental relations between the electron density distribution inside the sample, $\rho(\mathbf{r})$, and the observed scattering intensity, $I(\mathbf{s})$, are conveniently combined in a sketch

$$
\begin{aligned}
& \rho(\mathbf{r}) \stackrel{\mathscr{F}_{3}}{\Leftrightarrow} A(\mathbf{s}) \\
& z(\mathbf{r}) \underset{\Delta}{\Leftrightarrow} P(\mathbf{r}) \underset{\mathscr{F}_{3}}{\Leftrightarrow} I(\mathbf{s})
\end{aligned}
$$

from which the theoretically explored options for a quantitative analysis of the scattering, $I(\mathbf{s})$, can be accessed. According to the scheme, the real-space electron density, $\rho(\mathbf{r})$, by 3D complex Fourier transform is converted into the scattering amplitude, $A(\mathbf{s})$, in reciprocal space.

Stepping downward in the scheme from the amplitude, we arrive at the scattering intensity

$$
I(\mathbf{s})=|A(\mathbf{s})|^{2}
$$

by taking the square of the absolute value. The unidirectional downward arrow in Eq. (2.12) indicates that this operation cannot be reversed. We are losing the phase information of the structure. This means that we cannot reconstruct absolute positions of individual domains (i.e., crystallites) in the material. Only relative distances among domains, i.e., their correlations, can be determined. As a consequence of the last-mentioned operation, the intensity

$$
I(\mathbf{s})=I(-\mathbf{s})
$$

is always an even function (point symmetry) with real (not complex) values. In practice this means that we can reconstruct $I(\mathbf{s})$ from $I(-\mathbf{s})$ and vice versa if only one of these points has been measured on the detector (see for instance Fig. 2.3). Another consequence is relaxing the calculus, as long as we stay with the s-system: When switching back and forth between reciprocal and real space by means of the Fourier back-transform and the Fourier transform, even the switching sign in the harmonic kernel becomes negligible.

Proceeding to the left along the bottom edge of Eq. (2.12) we arrive back in real space at the Patterson function,

$$
P(\mathbf{r})=\mathscr{F}_{3}^{-1}(I(\mathbf{s})) .
$$

The physical meaning of the Patterson function is readily established by introduction and interpretation of the autocorrelation operation $\star 2$ 


$$
P(\mathbf{r})=\rho^{\star 2}(\mathbf{r}),
$$

which turns the structure $\rho(\mathbf{r})$ directly into $P(\mathbf{r})$. Because the autocorrelation integral expands into

$$
\rho^{\star 2}(\mathbf{r})=\int \rho(\mathbf{y}) \rho(\mathbf{r}+\mathbf{y}) d^{3} y,
$$

it is identified by the overlap integral between the structure, $\rho(\mathbf{y})$, and its displaced ghost. Here the vector $\mathbf{r}$ describes the amount and the direction of the displacement. In particular in the field of SAXS a common synonym for the Patterson function is the correlation function $[11,12]$,

$$
\gamma(\mathbf{r})=\rho^{\star 2}(\mathbf{r}) / \rho^{\star 2}(0)=P(\mathbf{r}) / \rho^{\star 2}(0) .
$$

By its normalization $\gamma(0)=1$ it indicates that the correlation between structure and ghost is perfect, if there is no displacement at all.

Finally, the structure of soft matter frequently may be considered to be made from domains which can be distinguished from each other by a sufficient difference of their electron densities (contrast). This is the case for materials comprising soft and hard domains, voids, crystallites, or amorphous regions. In this case it has proven advantageous not to study the correlation function, but to perform edge enhancement

$$
z(\mathbf{r})=\Delta P(\mathbf{r})
$$

by applying the Laplacian operator. A method for the computation of a 3D chord distribution function (CDF), $z(\mathbf{r})$, has been described in 2001 [13, 14], but before that a $1 \mathrm{D}$ chord distribution called interface distribution function (IDF), $g_{1}(r)$, had already been proposed by Ruland [15-17] for the study of lamellar systems. Moreover, the basic idea of this approach is reaching back to 1965, when Mèring and Tchoubar [18-21] devised the (radial) chord length distribution (CLD), $g(r)$.

\subsection{Options for an Analysis of the Scattering Intensity}

\subsubsection{Completeness - A Preliminary Note}

If the structure of an investigated sample can be approximated by a lattice (crystal), the result of the scattering experiment is a diffraction pattern with many distinct peaks - the Fourier transform of an infinite lattice is, again, an infinite lattice of peaks, and the success of crystallography is based on the fact that the lattice structure can completely be reconstructed from a limited number of diffraction peaks if only their positions and strengths are measured.

In general, the structure of soft matter is imperfect and polydisperse. Only in rare cases it can sufficiently be described by a crystal. Thus, in most of the practical cases, an in-depth analysis of scattering intensity by means of the crystallographic approach is not permitted. Although this fact is known for almost a century (Debye 
and Menke $^{7}$ (1931) [1]), far-reaching conclusions are still drawn, even if they are merely based on determinations of peak positions ("long periods") and peak shapes of scattering patterns.

Soft matter structure is characterized by a continuous density function $\rho(\mathbf{r})$, which is subjected to Fourier transform by the scattering experiment yielding a continuous intensity function $I(\mathbf{s})$ which does not drop to zero in long intervals between narrow peaks. Thus, for any in-depth analysis of distorted structure, either we have to model the complete shape of the pattern or we have to switch to real space, again supplying the transform by a complete intensity function.

\section{Example: Analysis of Ravel's Bolero}

Let us demonstrate the problem in analogy to music reproduced by a hi-fi system equipped with a spectrum analyzer display. As the playback of Ravel's Bolero, $\rho(t)$, has started we are watching the spectrum analyzer, which shows - in mathematical analogy to scattering $-I(v)=\mathscr{F}_{1}\left(\rho^{\star 2}(t)\right)$, the power spectral density of the music (Fig. 2.4). We interpret the spectrum by identifying a long period at $v \approx 200 \mathrm{~Hz}$ and a second one at $v \approx 200 \mathrm{kHz}$, interpreting the actual structure of the music by explaining that "the music is mainly made of two sinusoidal tones". In a more advanced approach we would consider the shape of $I(v)$, but because only a lo-fi system were available, we would only measure the low frequencies (in analogy to USAXS). After back-transformation we would be able to recognize drums playing and conclude that the piece is percussion. In contrary, we could as well use an instrument with a transmission at high frequencies (in analogy to SAXS) and would be led to the conclusion that the music is a piece for oboe and clarinet.



Fig. 2.4 The spectrum analyzer of a hi-fi system displays the power spectral density of the music, which is "the scattering intensity of the music", on a double logarithmic scale

\footnotetext{
${ }^{7}$ For a translation of the note made by Debye and Menke cf. [10], p. 1.
} 


\subsubsection{Analysis Options - Example for SAXS Data}

Options of data analysis can be deduced from Eq. (2.12) and our notions concerning the structure. As an example let us consider the case of small-angle X-ray scattering. In this area the structure is described by a continuous density function. Although there is no ${ }^{8}$ way back from intensity to density, there are several options for data analysis:

1. Utilize theory and find out how some structure parameters can be determined from the intensity directly;

2. walk from the intensity along the lower edge halfway back to real space, where the transformed data are closer to human perception;

3. model a structure and fit it to the intensity; or

4. in addition to item 2 carry out "edge enhancement" in order to visualize structure by means of the chord distribution function (CDF), $z(\mathbf{r})$, and interpret or fit it.

\section{Effort of Data Analysis}

The above-mentioned options are listed in the order of increasing complexity. When scattering curves (isotropic data) shall be analyzed, all the four listed options have proven to be manageable by many scientific groups.

In contrast, a real challenge is the analysis of scattering images from anisotropic materials, and in this subarea many scientists surrender and resort to the interpretation of peak positions and peak widths in raw data. So after having advanced by learning how to analyze curves, in the field of anisotropic materials we are presently in a similar situation as science has been in 1931 in respect to isotropic data.

A shortcut solution for the analysis of anisotropic data is found by mapping scattering images to scattering curves as has been devised by Bonart in 1966 [22]. Founded on Fourier transformation theory he has clarified that information on the structure "in a chosen direction" is not related to an intensity curve sliced from the pattern, but to a projection (cf. p. 37) of the pattern on the direction of interest.

A barrier to the application of the shortcut is probably resulting from the need to preprocess the scattering data and to project the 3D scattering intensity on a line. This task requires 3D geometrical imagination and knowledge of methods of digital image processing, a field that is quite new to the community of scatterers. Programmers, on the other hand, are rarely educated in the fields of scattering and multidimensional projections.

\subsubsection{Parameters, Functions, and Operations}

The scheme of Eq. (2.12) which is sketching the relation between structure and scattering intensity contains many parameters, functions, and operations which require explanation:

\footnotetext{
${ }^{8}$ Except for the case of anomalous SAXS.
} 
$[V]$ is the irradiated volume. It is defined by the sample thickness multiplied by the footprint of the incident primary beam on the sample

$[\rho(\mathbf{r})]$ the electron density (WAXS) or the electron density difference (SAXS)

$[A(\mathbf{s})]$ scattering amplitude

$\left[k=\rho^{\star 2}(0)\right]$ scattering power

$[Q=k / V]$ invariant

$[P(\mathbf{r})]$ Patterson function

$[\gamma(\mathbf{r})=P(\mathbf{r}) / k]$ SAXS correlation function

$[z(\mathbf{r})]$ chord distribution function $(\mathrm{CDF})$

The X-ray detector measures the intensity of electromagnetic waves, i.e., the absolute square ||$^{2}$ of their amplitude. Thus, in combination, the upper path between density and intensity through the square is written as

$$
I(\mathbf{s})=\left|\mathscr{F}_{3}(\rho(\mathbf{r}))\right|^{2} .
$$

In the lower path through the square we have an equivalent formulation

$$
I(\mathbf{s})=\mathscr{F}_{3}\left(\rho^{\star 2}(\mathbf{r})\right)
$$

with the Patterson or correlation function $\rho^{\star 2}(\mathbf{r})$ involved. $\rho^{\star 2}(\mathbf{r})$ is generated from the "inhomogeneities" $\rho(\mathbf{r})$ by means of the autocorrelation.

\subsection{From the Mathematical Laboratory of Scattering}

Favorable properties of the Fourier transform itself provide general means either to split the general problem of data analysis into sub-problems or even to obtain structure parameters without much modeling work. In this respect the Fourier slice theorem must be pointed out because of its superior impact on scattering (Bonart [22]; Baltá and Vonk [9], p. 15) and on several modern technologies. ${ }^{9}$ The theorem deals with projections and slices. It explains the weird information on structure that we retrieve if we study the scattering intensity cut from a pattern along a line that is extending outward from the center of the pattern. In fact, the respective intensity curve is called a slice (or a section). Last but not least, the theorem reveals an elegant way to overcome the recognized problem.

In combination with other important theorems of Fourier transformation theory many of the fundamental structural parameters in the field of scattering are readily established.

\footnotetext{
9 Computer tomography, magnetic resonance imaging, digital image processing, synchrotron micro-tomography [23, 24], 3D electron microscopy of block copolymers [25].
} 


\subsubsection{Convolution, Correlation, and Shape Functions}

Convolution and correlation are two mathematical operations which are almost the same. They are ubiquitous in all fields of science ranging from the social sciences ${ }^{10}$ over the natural sciences to practical applications in digital image processing (blurring). If a linear system is triggered by a non-ideal excitation function, its answer is not its natural transfer function, but the convolution of the excitation function and the transfer function which shall be determined. Thus, deconvolution (e.g., "unsharp masking" of a photo) is a common issue. An apparent problem in the field of scattering is the consideration of the influence of the real primary beam profile on the intensity $I(\mathbf{s})$ that would have been measured with an ideal primary beam of point-shaped cross section.

\section{Convolution}

The convolution of two 1D functions $f(r)$ and $g(r)$ is defined by

$$
\begin{aligned}
h(r) & =\int_{-\infty}^{\infty} f(y) g(r-y) d y \\
& :=f(r) \star g(r) .
\end{aligned}
$$

The definition of convolution is readily extended to the $n$-dimensional case.

\section{Correlation}

Similar to convolution the correlation operator is

$$
\begin{aligned}
h(x) & =\int_{-\infty}^{\infty} f(y) g(x+y) d y \\
& :=f(x) \otimes g(x)=f(x) \star g(-x) .
\end{aligned}
$$

If $f(x)$ and $g(x)$ are different, the integral is named cross-correlation. If both functions are identical, the integral is named autocorrelation. In the latter case we write

$$
h^{\star 2}(r)=h(r) \star h(-r)=\int_{-\infty}^{\infty} h(y) h(r+y) d y .
$$

In the field of scattering the autocorrelation is also known by the name "convolution square".

\footnotetext{
${ }^{10}$ For example, the result of an interview is the profile of the interviewer's questions convolved with the ideal response of the interviewed person.
} 
Shape functions

A shape function

$$
Y(\mathbf{r})= \begin{cases}1 & / \mathbf{r} \text { inside the region } \\ 0 & / \mathbf{r} \text { outside the region }\end{cases}
$$

describes a region. A region is, for example, a particle, a microfibril, a spherulite, the silhouette of a person on a picture. In digital image processing shape functions are named masks or regions of interest (ROI). Shape functions are the basic elements of topological structure both in the fields of scattering and diffraction. The Fourier transform of a shape function is denoted by

$$
\Phi(\mathbf{s})=\mathscr{F}(Y(\mathbf{r}))
$$

\subsubsection{The Slice}

Let $f$ be a deliberate function. It is defined in space, but there is good reason not to specify if it is the real or the reciprocal one. So let us denote the space by a vector $\mathbf{u}_{n}$. The index $n$ shall indicate that this vector has $n$ components, i.e., $\mathbf{u}_{n} \in \mathbb{R}^{n}$ is a member of the $n$-dimensional vector space of real numbers over which the function $f$ shall take its values. For application we will identify $\mathbf{u}_{n}$ either by $\mathbf{s}$ (and then the function $f$ may be the scattering intensity $I(\mathbf{s}))$ or by $\mathbf{r}$. Now let us consider a vector $\mathbf{u}_{m} \in \mathbb{R}^{m}$ that has less components $(m<n)$ than $\mathbf{u}_{n}$. We say $\mathbb{R}^{m}$ is a subspace of $\mathbb{R}^{n}$. This may be a plane or a line through $\mathbf{s}$-space or $\mathbf{r}$-space, respectively. There are many possible subspaces, but for the slice theorem to work we have to choose special subspaces which include the origin of the coordinate system. If we restrict the considered $n$-dimensional function $f\left(\mathbf{u}_{n}\right)$ to an $m$-dimensional subspace $\mathbf{u}_{m}$, we indicate this by writing $\lceil f\rceil_{m}\left(\mathbf{u}_{m}\right)$ and call this restricted function the $m$-dimensional slice (or section) of $f$ in the coordinates $\mathbf{u}_{m}$. Obviously, we can always rotate the coordinate system of $\mathbf{u}_{n}$ in such a way that the redundant coordinates $\mathbf{u}_{n-m}=0$ become zero. So the general mapping rule of a slice is

$$
\lceil f\rceil_{m}\left(\mathbf{u}_{m}\right)=\left.f\left(\mathbf{u}_{n}\right)\right|_{\mathbf{u}_{n-m}=0},
$$

with the vertical bar meaning "restricted to" or "at the position". So let us consider examples now. For a fiber scattering pattern $I(\mathbf{s})$ with the fiber axis rotated into $s_{3}$-direction,

$$
\lceil I(\mathbf{s})\rceil_{1}\left(s_{3}\right)=I\left(0,0, s_{3}\right)
$$

is an example for a 1D slice. It is a curve taken from the pattern along the meridian. A different slice is

$$
\lceil I(\mathbf{s})\rceil_{2}\left(s_{1}, s_{2}\right)=I\left(s_{1}, s_{2}, 0\right)=\lceil I(\mathbf{s})\rceil_{2}\left(s_{12}\right) .
$$


It is a $2 \mathrm{D}$ slice of the fiber pattern. Only fiber symmetry makes that it is completely represented by a curve as a function of a transverse (cf. Bonart [22]) coordinate $s_{12}=\sqrt{s_{1}^{2}+s_{2}^{2}}$ on the equator of the pattern.

Resorting to the definition of the Fourier transform, Eq. (2.7), we notice that for the redundant coordinates the harmonic kernel degenerates and becomes $\exp (0)=$ 1. Thus for the redundant coordinates the Fourier transform turns into a simple integration with respect to the respective reciprocal coordinate ${ }^{11}-\mathrm{a}$ "projection".

\subsubsection{The Projection}

As with the slice, the projection, as well, is mapping a function $f\left(\mathbf{u}_{n}\right), \mathbf{u}_{n} \in \mathbb{R}^{n}$, to a subspace. The mapping rule is

$$
\{f\}_{m}\left(\mathbf{u}_{m}\right)=\int f\left(\mathbf{u}_{n}\right) d \mathbf{u}_{n-m} .
$$

Thus we integrate $f$ over all those Cartesian coordinates from which the projected curve shall no longer be a function. Inexperienced scientists tend to make mistakes by simply "summing pixels" from the 2D scattering image collected on the detector, although the problem is from 3D s-space. So the question to answer first is, Is the information that I have gathered in my experiment complete? If the affirmative answer has been justified, the means of how the integration has to be performed are right at hand.

\subsubsection{Fourier Slice Theorem}

Under Fourier transform, slice and projection are exchanged and it follows

$$
\lceil h\rceil_{m}=\mathscr{F}_{m}\left(\{H\}_{m}\right),
$$

with $h(\mathbf{r})$ some function and $H(\mathbf{s})$ being its Fourier transform. The slice theorem is also known by the name central projection theorem.

\subsubsection{Fourier Derivative Theorem}

From the definition of Fourier transform the derivative theorem

$$
\mathscr{F}\left(\frac{d^{n} h(r)}{d r^{n}}\right)=(2 \pi i s)^{n} H(s)
$$

\footnotetext{
11 The respective reciprocal coordinate is called "dual coordinate" by the mathematician.
} 
is established by partial derivation. Extension to the multidimensional case is simple for even orders of the derivative [13]

$$
\begin{gathered}
\mathscr{F}\left(\nabla^{2 n} h(\mathbf{r})\right)=\left(-4 \pi^{2} s^{2}\right)^{n} H(\mathbf{s}) \\
\mathscr{F}(\Delta h(\mathbf{r}))=-4 \pi^{2} s^{2} H(\mathbf{s}),
\end{gathered}
$$

with $\nabla$ the gradient ("nabla") operator and

$$
\Delta=\sum_{i} \frac{\partial^{2}}{\partial u^{2}}
$$

the Laplacian - with the given definition valid in Cartesian coordinates.

\subsubsection{Breadth Theorem}

The integral breadth of a 1D, even, and Fourier-transformable function $h(r)$ is defined by

$$
B(h)=\frac{\int h(r) d r}{h(0)} .
$$

Then it follows from the slice theorem Eq. (2.25) for the integral breadth of the Fourier-transformed function $H(s)$

$$
B(H)=\frac{\int H(s) d s}{H(0)}=\frac{h(0)}{\int h(x) d x}=\frac{1}{B(h)} .
$$

In the field of scattering a simplified version of the Fourier breadth corollary Eq. (2.31) is known as the Scherrer equation. ${ }^{12}$ As a result, the inverse of the integral breadth of a peak or reflection is the size of the crystal in the direction perpendicular to the net planes that are related to the reflection.

In order to deduce Scherrer's equation first an infinite crystal is considered that is, second, restricted (i.e., multiplied) by a shape function (cf. p. 36). Thus from the Fourier convolution theorem (Sect. 2.5.9) it follows that in reciprocal space each reflection is convolved by the Fourier transform of the square of the shape function and Scherrer's equation is readily established.

\subsubsection{Dilation and Reciprocity}

From the definition of the Fourier transform it follows that

$$
\mathscr{F}\left(\frac{1}{a} h\left(\frac{r}{a}\right)\right)=H(a s) .
$$

${ }^{12}$ In the literature the Scherrer equation is frequently related to the full widths at half-maximum. This approximation is unnecessary. 
It is worth noting that $(1 / a) h(r / a)$ is the result of the dilation of $h(r)$ by the factor $a$ in which the area under the curve is conserved. The result in reciprocal space is a compressed function $H$. Applied to the discrete functions of crystallography, this property of the Fourier transform is known as Bragg's law.

\subsubsection{Dirac's $\delta$-Function}

A definition of Dirac's $\delta$-distribution is readily established from dilation. Let

$$
\int_{-\infty}^{\infty} h(r) d r=1
$$

be normalized. Then the dilated function

$$
\int_{-\infty}^{\infty} h\left(\frac{r}{a}\right) \frac{d r}{a}=1
$$

is still normalized and $\delta(r)$ is defined taking limits

$$
\delta(r)=\lim _{a \rightarrow 0} \frac{1}{a} h\left(\frac{r}{a}\right)
$$

whereupon the integral remains normalized.

\subsubsection{Convolution Theorem}

Under Fourier transform the convolution (Eq. (2.15)) is turned into a multiplication.

$$
\begin{aligned}
\mathscr{F}(f \star g) & =F G \\
\mathscr{F}(f g) & =F \star G .
\end{aligned}
$$

This property is readily established from the definition of Fourier transform and convolution. In many fields of science this theorem is utilized for the purpose of fast deconvolution after Stokes [26].

\subsubsection{Bandlimited Functions}

If, in practice, a Fourier transformation shall be carried out, it is meaningful to search for functions that are not only bounded, but which even vanish when taking limits $|\mathbf{s}| \rightarrow \infty$ or $|\mathbf{r}| \rightarrow \infty$. Such functions are called bandlimited. Let us consider the function $h(\mathbf{r})=\mathscr{F}(H(\mathbf{s}))$. Then the reciprocal space image $H(\mathbf{s})$ is bandlimited if its Fourier transform, $h(\mathbf{r})$, does not contain spatial frequencies above a value of $f_{u}$, i.e.,

$$
h(\mathbf{r})=0 \text { for }|\mathbf{r}|>f_{u},
$$


and $f_{u}$ is the upper frequency of the frequency band. In mathematics, band limitation is expressed in terms of functions with "finite support". The support

$$
\operatorname{supp}(h)
$$

of the function $h$ is the region in which the function $h(\mathbf{r})$ does not vanish.

\section{Revisiting the Analysis of Ravel's Bolero}

Let us finish our demonstration of the mathematical toolbox of scattering theory by revisiting the example of the analysis of Ravel's bolero on p. 32. If the power spectral density of the music, indeed, would have missed the high frequencies, the "USAXS" analysis based on only the low frequencies would have been correct. In other words, if discrete scattering is only observed in the USAXS regime, we can subtract the diffuse background and keep the complete, bandlimited structure information for further analysis. On the other hand, if the discrete scattering of our material is extending out into the SAXS region, the experiment has to be carried out with much higher effort. For the sake of completeness we will measure the SAXS pattern and will deconvolve it from its relatively broad primary beam. Then we will measure the USAXS pattern and combine it with the deconvolved SAXS pattern before a quantitative analysis is performed.

\subsubsection{Deconvolution}

At the first glance it appears intriguing to carry out a fast deconvolution by application of the convolution theorem Eq. (2.36). After Fourier transform of both the measured intensity $I_{m}(\mathbf{x})$ and the measured primary beam profile $W_{m}(\mathbf{x})$ the pointfocus intensity

$$
I(\mathbf{x})=\mathscr{F}_{2}^{-1}\left(\mathscr{F}_{2}\left(I_{m}(\mathbf{x})\right) / \mathscr{F}_{2}\left(W_{m}(\mathbf{x})\right)\right)
$$

is retrieved from the back-transformation of their ratio. Here $\mathbf{x}$ is the vector in the detector plane which addresses the pixels of the recorded pattern. In practice [27], a major problem results from the division of two bandlimited and noisy functions. Thus, the division causes extreme amplification of noise, and a cumbersome adjustment of a digital low-pass filter is required, which is prone to distort $I(\mathbf{x})$. The sensitivity of this approach can easily be tested by a rapidly written computer program, if a scientific signal processing programming environment like, e.g., pv-wave ${ }^{\circledR}[28]$ or IDL ${ }^{\circledR}[29]$ is available.

More convenient is the iterative approach, as devised by v. Cittert [30-32]. Although the iterative method takes more time (typically 1 min until convergence is reached), it has an important advantage, because convergence means that the result is correct. As has been proposed in the field of X-ray scattering by Glatter [32], the intrinsic correction function which is computed in each iterative step should be smoothed. Consulting the programmer's manual of a modern signal processing tool 
[28], this task is no longer performed by convolution with a triangle or cone [32], but by application of an adapted smoothing digital filter. Finally, it should be mentioned that the introduction of cyclic boundary conditions to the measured intensity is a fast solution that prevents from artifacts, which otherwise are creeping into the detector plane from its edges while the iteration is proceeding.

\subsection{Application to Materials with Fiber Symmetry}

\section{Overview}

Materials with fiber symmetry show an anisotropic scattering pattern. The analysis of isotropic scattering is not addressed here, because it has been disseminated in many textbooks $[9,10,33,34]$ and is, in general, less ambitious than the analysis of anisotropic materials.

\subsubsection{Isotropization and Azimuthal Averaging}

If a measured scattering pattern shows fiber symmetry, it is sometimes of interest to compute the corresponding isotropic pattern in order to apply well-known algorithms that are adapted to the analysis of isotropic data. Azimuthal averaging is not a solution, because it does not consider the 3D nature of the fiber pattern in reciprocal space. On the other hand, the error introduced by azimuthal averaging can be tolerated if the observed anisotropy is small.

\section{Isotropization}

In an exercise aiming at the isotropization of a fiber pattern, $I\left(s_{12}, s_{3}\right)$, with the fiber axis $s_{3}$ one may decide to, first, project the observed intensity on the fiber axis

$$
\{I\}_{1}\left(s_{3}\right)=2 \pi \int_{0}^{\infty} s_{12} I\left(s_{12}, s_{3}\right) d s_{12},
$$

meaning that each column of the intensity matrix is multiplied by $2 \pi s_{12}$, the circumference of a circle of radius $s_{12}$ - the distance of the column from the fiber axis (meridian). The result is Bonart's longitudinal scattering [22]. Although from this result the isotropic intensity $I(s)$ can be computed [6, p. 606-607], it requires some computational effort. In practice, it is more convenient to proceed as described in my textbook [10, p. 131] and to write a program for the compact solution

$$
I(s)=\frac{1}{s} \int_{0}^{\pi / 2} s_{12} I\left(s_{12}, s_{3}\right) d \phi,
$$


which is understood as a three-step operation. First, each column of the fiber pattern is multiplied by its distance from the fiber axis. Second, an azimuthal average is performed. Third, the result is divided by $s$.

\section{Azimuthal Average in Practice}

The integration about the azimuthal angle $\phi$ which is part of the isotropization routine is a frequent problem in image processing in the Fourier space. Thus, scientific application development systems like pv-wave ${ }^{\circledR}$ [28], IDL, or MatLab provide special programming constructs which ease the realization of such algorithms. The special library functions used in this example are DIST, SHIFT, and WHERE. The meaning can be looked up in the reference manuals of pv-wave or IDL, which can be found on the Internet.

The second example on projection should be easier to understand for the novice.

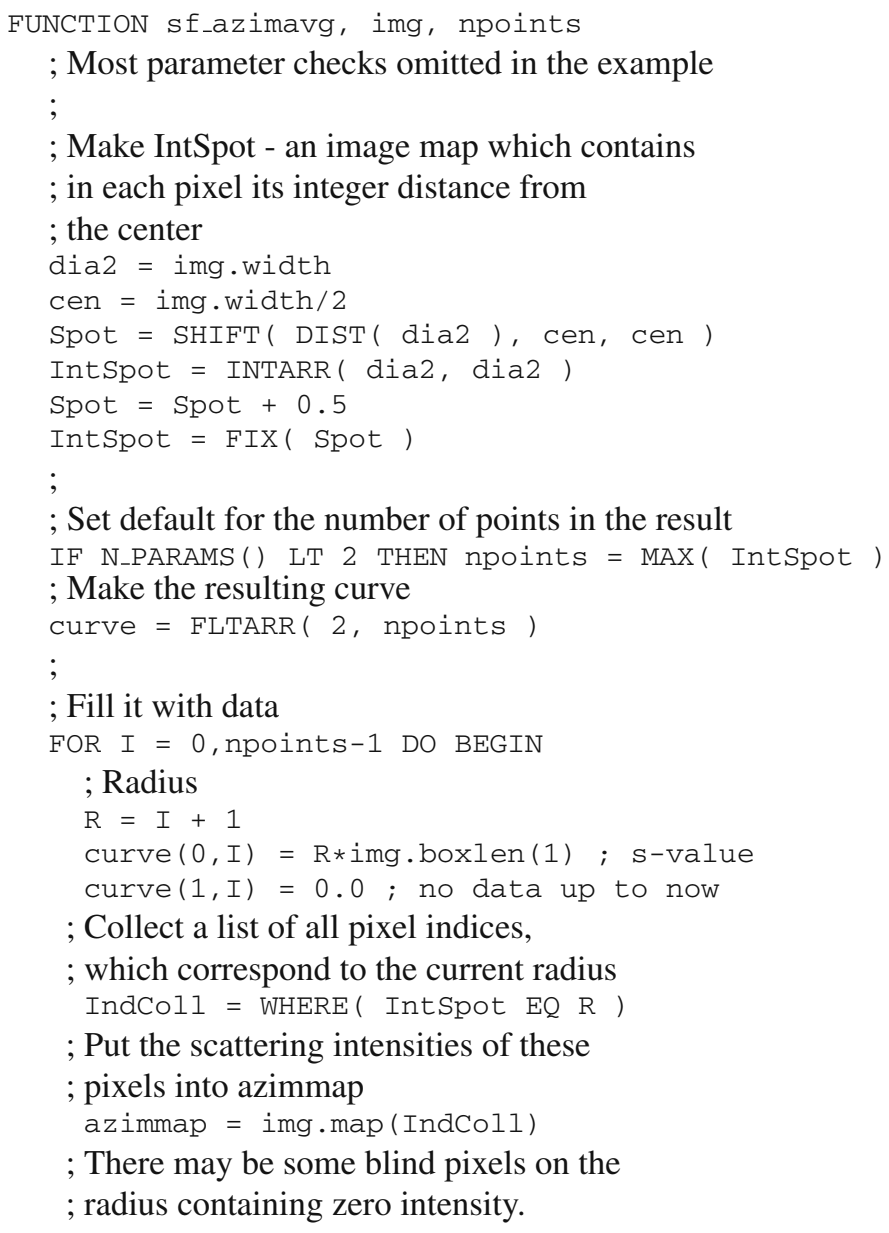


; Extract a list of only the good ones!

GoodColl = WHERE ( azimmap GT 0.0 )

; Are there any good ones at all?

; Otherwise there is nothing to average

IF GoodColl(0) GE 0 THEN BEGIN

; Extract a list of only the valid pixels

goodmap = azimmap (GoodColl)

; and store the average of the list

; in the scattering intensity

curve $(1, I)=$ AVG ( goodmap )

ENDIF

ENDFOR

RETURN, curve

END ; Procedure sf_azimavg

\section{Projection in Practice}

Utilizing pv-wave ${ }^{\circledR}[28]$ it takes only a few lines of programming code to implement the already mentioned projection of a fiber pattern on the meridian, provided the image data are stored in a convenient data structure. The result of this function is the longitudinal scattering of the fiber pattern, as introduced by Bonart [22]. The following listing shows the corresponding source code.

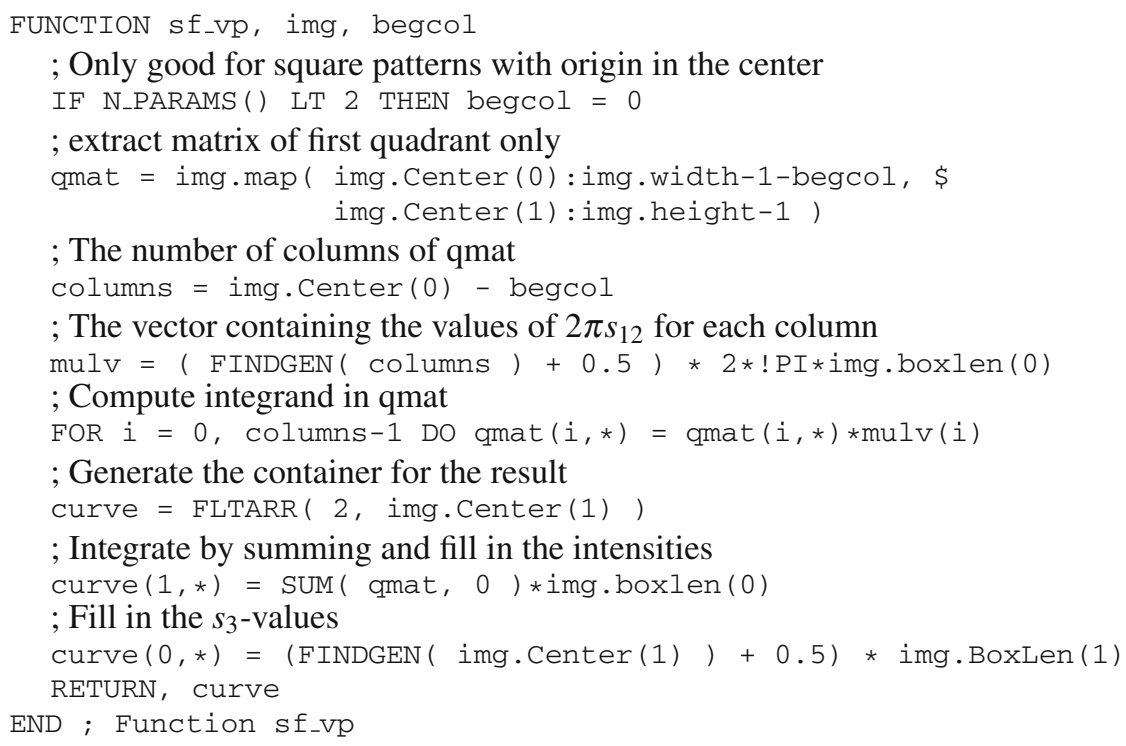

The function is called with two parameters, the first parameter img is the image (comprising the map of the scattering intensities and all the information that is essential for a scattering pattern). The second parameter begcol tells the program in which column to start with the integration. This may be useful if the image contains 
a blind region around the valid pixels. Comments start with a semicolon, a $\$$ sign at the end of a line indicates that the statement is continued on the next line.

The first line of code inside the function ensures that begcol gets a default value (assuming that all the pixels of the map contain valid data).

qmat is the upper left quadrant of the scattering pattern img . map which shall be processed. columns is assigned the number of valid columns in the image which reach out from the center. img. Center $(0)$ contains the x-coordinate of the center, img. Center (1) contains the y-coordinate. img.boxlen (0) and img.boxlen (1) contain the horizontal and the vertical size of each pixel, i.e., $\Delta s_{12}$ and $\Delta s_{3}$, respectively, in units of reciprocal nanometers. These definitions are part of the conceptual design. ${ }^{13}$

mulv is a vector which contains the distance of each column from the center multiplied by $2 \pi s_{12}$. The following FOR loop makes the integrand by multiplying each column of qmat by the corresponding element of mulv.

Now we need a container for the result. The result is a curve, so we define it by a matrix of two columns and the same number of rows as qmat. The column 0 is for the $s_{3}$-values, column 1 for the values of $\{I\}_{1}$. Because qmat has already been prepared for a "usual" integration, the operator SUM of pv-wave can be used to compute the numerical integral, the index "*" indicating that the respective operation has to be performed for all pixels in the respective direction of the matrix. Finally, the $s_{3}$-values are filled in, and the result is returned.

The typical call of the function sf_vp would be issued by C=sf_vp ( scatpat) - if scatpat would have been loaded with a scattering pattern which has suitably been preprocessed [10]. The result can be inspected by PRINT, c. It can be plotted by sf_cplot, $c$. It can be stored in a file by issuing sf_cwrite, c, 'myfile.dat', if my programs sf_progs.tar.bz2 have been downloaded [35].

\subsubsection{Analysis by Study of Projections}

The basic principle of the methods presented in this section is the extraction of curves from the $2 \mathrm{D}$ data in the scattering patterns by projection integrals. The importance of such projections for structure analysis in scattering patterns has early been recognized by Bonart [22]. In fact, the invariant $Q$, which is frequently studied in the field of X-ray scattering, is nothing but the projection

$$
Q=\int I(\mathbf{s}) / V d^{3} s
$$

of the scattering intensity normalized to absolute values, ${ }^{14} I(\mathbf{s}) / V$, on the origin of reciprocal space. The method of how to interpret $Q$ is exemplified in many textbooks of scattering $[9,10,34]$.

\footnotetext{
${ }^{13}$ The conceptual design of my program suite is defined in the file sfa_structure.pro

14 The absolute intensity is expressed in e.u. $/ \mathrm{nm}^{3}$, with e.u. meaning electron units.
} 
By suitable projection of the 2D image data onto a line (longitudinal scattering, first method) or onto a plane (transverse scattering, second method) scattering curves are extracted, which finally can be analyzed in terms of two-phase structural models considering soft domains and hard domains.

In ignorance of scattering theory (cf. Sect. 2.5.4 Fourier Slice Theorem), curves are frequently cut (sliced) from scattering patterns (reciprocal space) and analyzed in terms of materials structure (real space).

\subsubsection{1D Projections}

While the 0D projection is only a number, a 1D projection on direction $s_{i},\{I\}_{1}\left(s_{i}\right)$, is a curve. Thus, some of the pre-evaluation steps that necessarily must be performed before computation of $Q$ (diffuse background subtraction, determination of Porod's law [10]) may be deferred to be operated later on the curve. Particularly useful in materials science is a special 1D projection: the projection of a fiber pattern on the fiber axis, $s_{3}$ (cf. Eq. (2.40)) - Bonart's longitudinal scattering. The listing of a corresponding computer program starts on p. 43. Application of the Fourier Slice Theorem (Sect. 2.5.4) yields

$$
\lceil P\rceil_{1}\left(r_{3}\right)=\mathscr{F}_{1}\left(\{I\}_{1}\left(s_{3}\right)\right)
$$

i.e., the projected scattering intensity is related to the slice of the Patterson function (correlation function) in direction $r_{3}$.

\section{General Information Content}

Thus, the topological information contained in a 1D projection of the scattering intensity for a two-phase system ${ }^{15}$ is readily demonstrated in Fig. 2.5. By choosing a projection direction in the fiber pattern, the respective direction in the materials structure is selected (indicated by a double arrow in Fig. 2.5). Now we draw straight lines ("chords") only in the chosen direction through the structure which penetrate the two-phase or multiphase structure. The edges of the domains cut these chords into segments, and the distributions of segment lengths which are present in the material in the chosen direction are generating $\{I\}_{1}\left(s_{i}\right)$. Not only single segments are contributing, but also combinations of adjacent segments. In this respect broken lines in the sketch indicate some combined segment lengths that are contributing to the average long period.

\footnotetext{
15 The two phases may be hard domains and soft domains, respectively - or crystalline and amorphous domains.
} 

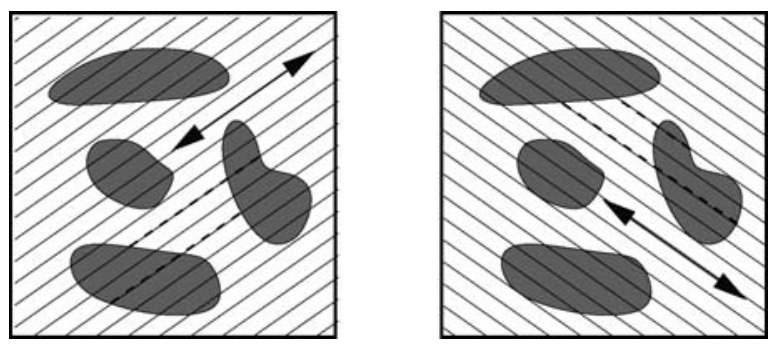

Fig. 2.5 The topological information on the structure of a multiphase system that is related to 1D projections $\{I\}_{1}\left(s_{i}\right)$ in different directions. The demonstration shows two directions indicated by arrows and the related chords. From $\{I\}_{1}\left(s_{i}\right)$ the distributions of the chord segments between domain edges are retrieved. Long periods are indicated by broken lines

\section{Relation to Lamellar Stack Systems}

Traveling along a chord, we will alternately move through the hard and the soft domain phase, from time to time crossing a phase boundary. Thus structural parameters of physical interest are $\bar{d}_{h}$, the average travel distance inside the hard domain phase, $\bar{d}_{s}$, the average travel distance inside the soft domain phase, as well as the variances $\left(\sigma_{h}\right.$, and $\left.\sigma_{s}\right)$ of their distributions, which shall be called "domain height distributions" $h_{h}\left(r_{3}\right)$ and $h_{s}\left(r_{3}\right)$.

Thus, by computing the longitudinal structure we eliminate all information on transverse correlation of domains from the scattering pattern and reduce the problem to the case of 1D scattering curves, which is well known from the theory of lamellar two-phase systems. For the solution of the 1D problem appropriate data analysis methods are at hand [15, 36-38].

Analysis of isotropic samples with a lamellar domain structure and the analysis of longitudinal intensity projections are closely related to each other. In the first case a Lorentz correction $\left(I_{1}\left(s_{3}\right)=2 \pi s_{3}^{2} I\left(s_{3}\right) ; s_{3} \equiv s\right)$ and in the second case a projection result in an intensity which is related to a $1 D$ structure in physical space. Thus, after the extraction of the "1D intensity", analysis proceeds in the same way for both cases. While from a mathematical point of view the first method is only applicable for isotropic samples with a lamellar structure, the second is generally applicable for two-phase or even multiphase topologies. From the physical point of view the second method is favorably applied to well-oriented systems. Both kinds of 1D intensities share the same 1D Porod's law falling off with $s_{3}^{-2}$, because they both are related to sections in physical space. Generally, comparing projection analysis to an analysis of intensity sections, there is no dilemma concerning the expected falloff of scattering intensity in the Porod region with projections even in the case of varying misorientation. Finally, the well-known dilemma with intensity sections is only a result of the unknown effect of the projection operation which is awkward in the space where the structure is.

The method has been applied in several experimental studies [37, 39-47] in which either the intensity projection is computed, transformed to real space, and 
finally analyzed by means of a 1D statistical model or in which the fiber pattern is transformed to real space as a whole (cf. Sect. 2.6.3), before the longitudinal structure information is sliced from it and analyzed.

\subsubsection{2D Projections}

A classical device that performs a 2D projection of the scattering pattern is the Kratky camera. By integrating the intensity along the direction of the (infinitely) long focus slit, it is collapsing the SAXS intensity on the plane that is normal to the slit direction. This mechanism is the general feature of $2 \mathrm{D}$ projections. As in the case of the $1 \mathrm{D}$ projections, the orientation of the plane may be chosen deliberately. The result of such a projection $\{I\}_{2}\left(s_{j}, s_{k}\right)$ is not a curve as was the case with the $1 \mathrm{D}$ projection, but a $2 \mathrm{D}$ scattering pattern. Only in the case of $2 \mathrm{D}$ isotropy within this plane the 2D scattering intensity is fully described by a curve taken along the radius. This $2 \mathrm{D}$ isotropy is fulfilled for materials with fiber symmetry, if the intensity is projected on the equatorial plane

$$
\{I\}_{2}\left(s_{12}\right)=2 \int_{0}^{\infty} I\left(s_{12}, s_{3}\right) d s_{3}
$$

The resulting intensity describes Bonart's [22] transverse structure of the fiber - the arrangement of domain cross-sections in the fiber cross section.

In a study of a poly(ether ester) material [48], the evolution of its transverse structure during straining in a stretch-hold [49] experiment has been evaluated. $\{I\}_{2}\left(s_{12}\right)$ is computed by means of a program which is much more simple than $\mathrm{sf}$-vp.pro listed on p. 43.

As is known from the historic Kratky camera technique [34, 50], $\{I\}_{2}\left(s_{12}\right)$ exhibits a Porod's law with the scattering falling off with $s_{12}^{-3}$. Small deviations from the predicted falloff are accounted to the non-ideal structure of the real two-phase system [51, 52] and corrected accordingly. Thereafter the 2D interference function $G_{2}\left(s_{12}\right)$ of an ideal two-phase system

$$
G_{2}\left(s_{12}\right)=\left(\left\{I_{2}\left(s_{12}\right)-I_{F l}\right) s_{12}^{3} / \exp \left(-\frac{4}{9} \pi^{2} d_{z}^{2} s_{12}^{2}\right)-A_{P_{2}}\right.
$$

is computed. ${ }^{16} A_{P_{2}}$, Porod's asymptote of the projected SAXS intensity, is the constant governing Porod's law. The non-ideal structure of the real two-phase system is described by $I_{F l}$ and $d_{z}[10,51]$. Fluctuations of the electron density are considered by $I_{F l}$, the density fluctuation background. $d_{z}$ is the width of the transition zone at the domain boundary.

\footnotetext{
${ }^{16}$ It appears worth to be noted that this and all other kinds of interference functions share the favorable properties of bandlimited functions (cf. Eq. (2.38) on p. 39).
} 
From $G_{2}\left(s_{12}\right)$ the $2 \mathrm{D}$ chord distribution $[21,53] g_{2}\left(r_{12}\right)$ is computed by 2D Fourier transform in plane polar coordinates, ${ }^{17}$

$$
g_{2}\left(r_{12}\right)=2 \pi r_{12} \int_{0}^{\infty} \mathrm{J}_{0}\left(2 \pi r_{12} s_{12}\right) G_{2}\left(s_{12}\right) d s_{12}
$$

Here $\mathbf{J}_{0}$ denotes the Bessel function of the first kind. In general, $g_{2}\left(r_{12}\right)$ shows the distribution of chords from needle and matrix cross sections and their correlations in the plane normal to the fiber direction.

In the analysis of the experimental data it turns out that $g_{2}\left(r_{12}\right)$ is positive everywhere. Thus the correlations among the "disks in the plane" are negligible, and $g_{2}\left(r_{12}\right)$ represents the chord distribution of an ensemble of uncorrelated disks in the $\left(r_{1}, r_{2}\right)$-plane. Following the principle of late modeling, now it appeared reasonable to model the cross section of every needle by a circular disk and to ask for the properties of a needle diameter distribution, $h_{D}(D) . g_{2}\left(r_{12}\right)$, which can be expressed in terms of $h_{D}(D)$ and the intrinsic chord distribution $g_{c}\left(r_{12}\right)$ of a disk with unit diameter

$$
g_{2}\left(r_{12}\right)=\int_{0}^{\infty} h_{D}(D) g_{c}\left(\frac{r_{12}}{D}\right) \frac{d D}{D},
$$

as simply the superposition of compressed and expanded images from $g_{c}$ weighted by the value of the diameter distribution, $h_{D}(D)$, which shall be studied. Equation (2.46) is the definition of the Mellin convolution [36, 54]. $h_{D}(D)$ can be computed by numerical inversion of Eq. (2.46) utilizing an iterative v. Cittert algorithm similar to the one proposed by Glatter [55]. Instead of doing so, one can take advantage of the special mathematical properties of the Mellin convolution and directly compute parameters characterizing the needle diameter distribution from the measured chord distribution utilizing moment arithmetic [48]. Figure 2.6 shows transverse scattering curves, $\{I\}_{2}\left(s_{12}\right)$, computed from the fiber patterns of the elastomer as a function of elongation, $\varepsilon$, and the corresponding $2 \mathrm{D}$ chord distributions, $g\left(r_{12}\right)$. It appears worth to be mentioned that the transformation to real space is carried out without any need to model the structure. Simple inspection reveals that the curves are positive everywhere in good approximation. This means that correlations among the microfibrils in transverse direction can be neglected. Assuming microfibrils of circular cross section, now the curves can be analyzed quantitatively. Figure 2.7(a) shows the diameter distributions of the microfibrils as a function of elongation as computed by Mellin deconvolution. Figure 2.7(b) shows parameters of the nanostructure computed directly from the $2 \mathrm{D}$ chord distributions by application of moment arithmetic: the average needle diameter, $\bar{D}$, the relative width of the needle diameter distribution, $\sigma / \bar{D}$, and the total cross section of the needles with respect to the total cross section of the fiber. Figure 2.7(b) shows that the mean diameter of the needle-shaped domains decreases almost linearly with increasing elongation, while for rubber elastic behavior, one would have expected a decrease according

\footnotetext{
${ }^{17}$ The simplification of the equation with respect to the version published in the references follows from general mathematical treatment of isotropization of the Fourier kernel in deliberate dimensions, as demonstrated by C. Burger in private communication.
} 


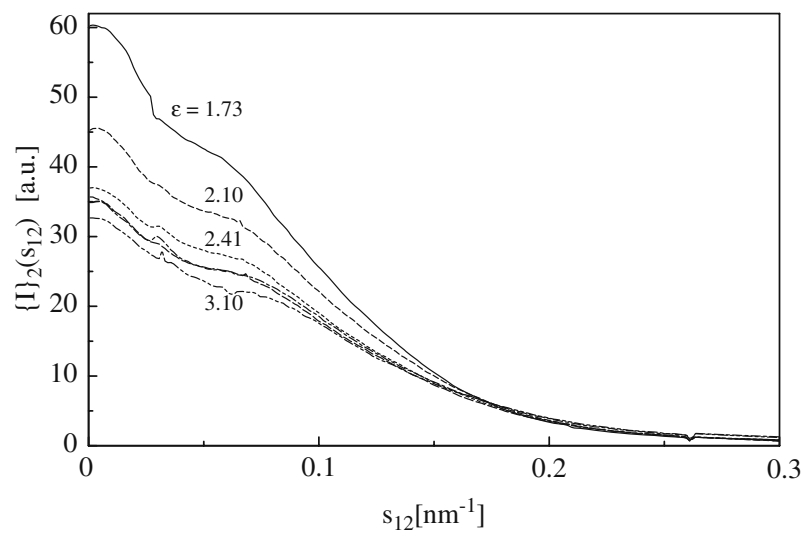

(a)



(b)

Fig. 2.6 (a) Projections $\{I\}_{2}\left(s_{12}\right)$ of the equatorial scattering onto the plane normal to straining direction. Arnitel E2000/60. (b) Chord distributions $g_{2}\left(r_{12}\right)$ of Arnitel E2000/60 computed from the curves in (a)

to $\bar{D}(\varepsilon)=\bar{D}_{0} / \sqrt{\varepsilon+1}$. As demonstrated in Fig. 2.7(a), the reason is that the disk diameter distribution alters its shape. With increasing elongation more and more thin needles are emerging, which cause the average diameter to decrease considerably. $\sigma / \bar{D}(\varepsilon)$, the relative width parameter of the disk diameter distribution, hardly increases. The total needle cross section per fiber cross section becomes constant

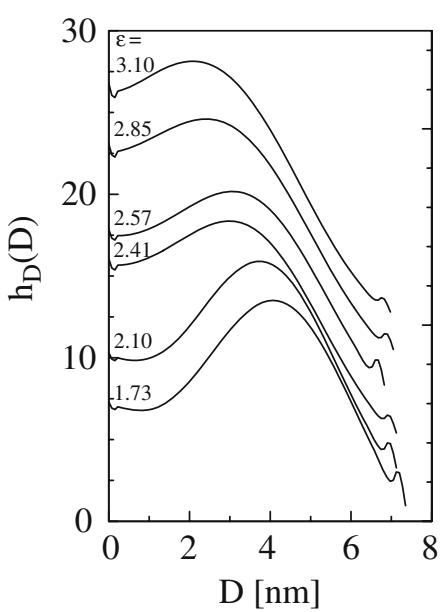

(a)

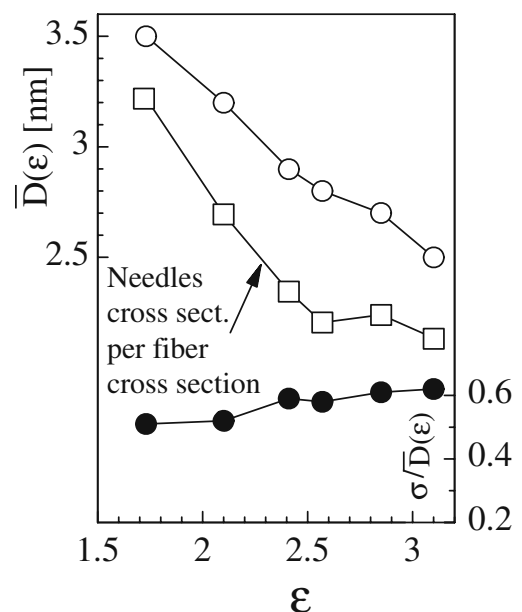

(b)

Fig. 2.7 (a) Diameter distributions, $h_{D}(D)$, of soft domain needles computed by numerical Mellin deconvolution of curves shown in Fig. 2.6. (b) Characterization of the ensemble of needle-shaped soft domains in Arnitel E2000/60 as a function of elongation $\varepsilon$ 
for elongations $\varepsilon>2.5$. On the other hand, in the observable region of elongations $1.7<\varepsilon<2.5$ a considerable decrease is observed. This decrease indicates a strain hardening process of the soft needles: During the straining process the soft material of the needles is compressed in transverse direction with respect to the surrounding matrix material. An assumed increase of the needle density during this process would amplify the observed effect. The measured values could be compared with measurements of Young's modulus, and stress-induced polymorphic transitions [56] could be discussed in conjunction with the presented result. For comprehensive discussion of the theoretical background and the results see the original paper [48].

\subsubsection{Visualization of Nanostructure from SAXS Fiber Patterns}

With the advent of fast, 2D detectors it has become possible to record anisotropic scattering patterns with cycle times of seconds only. New challenges are related to this technique.

First, there is no theory for anisotropic patterns that describes how to subtract the diffuse background. In the preceding section an intermediate solution has been presented, namely the projection of scattering data followed by data analysis of the resulting curve. The analysis of these curves is well-founded in theory and the treatment closely resembles the analysis of isotropic scattering. Nevertheless, it is cumbersome, time consuming, and may be biased because of the manual processing that is required. Second, the processing of voluminous series of $2 \mathrm{D}$ scattering patterns can only be managed by an automatic algorithm. Third, an interpretation of the observable complex scattering patterns by means of notions either derived from crystallography (e.g., Bragg's law) or derived from models adapted to the low information content of isotropic scattering (e.g., paracrystalline lattice) appears to be inadequate with respect to the apparently high information content of the recorded data.

\subsubsection{Projection on the Representative Plane}

Bearing in mind that for the analysis of real-space structure we are interested in a multidimensional image but not in a projection ${ }^{18}$ of the structural image, in reciprocal space we have to perform a projection ${ }^{19}$ on the representative plane $\left(s_{1}, s_{3}\right)$ of the fiber pattern (cf. Fig. 2.2) and compute

$$
\{I\}_{2}\left(s_{1}, s_{3}\right)=\int I\left(s_{12}, s_{3}\right) d s_{2} .
$$

\footnotetext{
18 This is in perfect analogy to an example from the area of medicine: We are not satisfied with a "chest X-ray", we want to study the tomogram. So the appropriate technique is backprojection.

${ }^{19}$ Application of the Fourier slice theorem (Sect. 2.5.4), the fundament of tomography.
} 
This evaluation step has two beneficial effects. First, it converts the related visualization of structure from a smeared (projected, "chest X-ray") to a clear ("tomogram") one. Second, it reduces noise in the pattern because of the averaging nature of the integral. Thus, we can afford to reduce the exposure during experiment and increase the time resolution of a structure evolution study. The source code of this projection is the most involved of all the projections that we are using.



; Make underlying matrix of $s_{12}$-values in scatterers paradigm: Center of the pattern is

; the corner shared by the four central pixels.

half = primg.center $(0)$

hor $=\operatorname{SHIFT}($ DINDGEN (primg $\cdot$ width $)+0.5$, half $)$

; The left wing of hor is not yet reflecting the distance of the pixel from its center hor $(0:$ half-1) = primg.width - hor $(0:$ half-1 $)$

; make a unit column vector

ver $=$ DBLARR (primg.height) +1

; and generate $s_{12}$ by matrix product

s12 = hor \# ver

$\mathrm{s} 12 \mathrm{q}=\mathrm{s} 12^{\wedge} 2$; The matrix of $s_{12}^{2}$-values

; Let us integrate on the left hand side only.

; Number of columns on the left side:

columns $=$ primg. Center $(0)-1$

truecen $=$ primg. center $(0)-0.5$

; *** Compute the integral for every column on the left hand side

FOR I $=1$, columns DO BEGIN

; for the I-th column (coordinate: $s_{1}$ ) there are I columns on the left

; of it which must be processed (in $s_{12}$ ). Cut out these columns!

is $12=\operatorname{data}(0: I-1, *)$

; compute the variable substitution matrix $d s_{2}$ in del

del $=\operatorname{si2q}(0: I-1, *)-(I-\text { truecen })^{\wedge} 2$

del $=\operatorname{si2}(0: I-1, *) / \operatorname{SQRT}(\mathrm{del})$

; now del is $s_{12} / \sqrt{s_{12}^{2}-s_{1}^{2}}$

; del goes with $1 / \sin \phi$, with $\phi$ being the angle between $s_{12}$-direction

; and $s_{1}$-axis, and this poses a singularity problem on the numerical integration to be

; carried out. Multiply intensities by line element $d s_{2}$

is $12=i s 12 *$ del

; Sum $I s_{12}$ over its first dimension into projected column

; In order to circumvent the singularity, the integral range is 1.5

; pixels shorter than the required overall length - we will take

; care for the special value later in the last statement of the loop.

summe $=\operatorname{SUM}($ is 12,0$)$

primg.map $(I, *)=$ summe $+1.5 * i . s 12(I-1, *)$ 
ENDFOR ; project over all the columns on the left ; copy from left (projected) to right side of the result $\mathrm{col}=$ columns +1 primg.map $(\operatorname{col}: 2 * \operatorname{col}-1, *)=\operatorname{ROTATE}(\operatorname{primg} \cdot \operatorname{map}(0: \operatorname{col}$ umns, $*), 5)$

; $* * *$ End projection $* * *$

RETURN, primg

END ; Function sf_fibproj

\subsubsection{Laplacian and Background Correction}

The next step of a proposed solution [13] extracts the discrete scattering from the pattern which has already been subjected to the Laplacian in real space (cf. Eq. (2.12)). For this purpose, first an estimated density fluctuation background is subtracted which is computed from the data points outside the inscribed circle of the image by the definition

$$
I_{F l}\left(s_{12}, s_{3}\right)=c_{0}+c_{12} s_{12}^{2}+c_{3} s_{3}^{2} .
$$

This evaluation step conforms to the rule that the density fluctuation background is expanded in even powers of the scattering vector. After the subtraction, the remnant discrete intensity is multiplied by $4 \pi^{2} s^{2}$. This multiplication is equivalent to the Laplacian ${ }^{20}$ edge-enhancement operator, as is evidenced by double application of the derivative theorem (Sect. 2.5.5).

Now a correction for the non-ideal character of the multiphase topology must be carried out. In the classical analysis this is a manual evaluation step which results in the subtraction of a slowly varying background [10, 51, 57]. Here the background determination is replaced by the result of a low-pass filter, applied to the scattering image at the present state of evaluation. The following listing shows the skeleton of a function for pv-wave or IDL which carries out the filtering of the scattering pattern img.

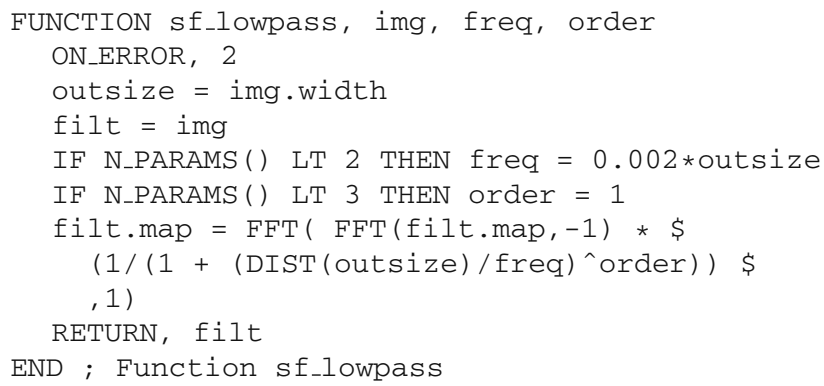

Expressing the cutoff frequency in relative units of the bandwidth (in the listing a default value of 0.002 , i.e., $0.2 \%$, is implemented), a filter is defined which is

\footnotetext{
${ }^{20}$ Conceded, the negative sign is missing - but we hate to work with negative intensities and will take care of the sign later.
} 
good for general use. The statement which assigns intensities to the resulting image matrix filt.map is the definition of a Butterworth filter.

\subsubsection{Fourier Transform}

After background subtraction, an interference function $G_{2}\left(s_{1}, s_{3}\right)$ is obtained and its 2D Fourier transform,

$$
z\left(r_{1}, r_{3}\right)=-\mathscr{F}_{2}\left(G_{2}\left(s_{1}, s_{3}\right)\right)
$$

is the multidimensional chord distribution. Because of fiber symmetry, the plane $\left(r_{1}, r_{3}\right)$ is representative for the $3 \mathrm{D}$ structure, and it is permitted to identify $r_{1} \rightarrow r_{12}$. Thus, $z\left(r_{12}, r_{3}\right)$ has been computed.

\subsubsection{Interpretation of the CDF}

\section{Fundaments and Final Goal}

Interpretation of this function is possible by means of the notions developed for the analysis of the respective radial chord length distribution (CLD), $g(r)$, by Méring and Tchoubar [18, 20, 21, 58] and by the notions deduced for the analysis of the 1D interface distribution function (IDF) developed by Ruland $[15,16]$ for the study of lamellar and microfibrillar systems.

The resulting function clearly exhibits the fundamental structural features of the nanostructure, and its evolution is observed in time series. Thus, in principle adapted structure models may be built which can serve the purpose of full quantitative analysis of the data. Unfortunately, the results show such a high complexity of the nanostructure that the programming of such a model appears to be extremely involved. Until the time when such 3D models will be available, the problem can be reduced by focusing on models only for either the longitudinal or the transverse structure, which finally can be analyzed quantitatively.

\section{Properties}

As has been exemplified in the description of its computation, the fiber-symmetrical $\mathrm{CDF}$

$$
z\left(r_{12}, r_{3}\right)=\left(\nabla \rho\left(r_{12}, r_{3}\right)\right)^{\star 2}=k \Delta \gamma\left(r_{12}, r_{3}\right)
$$

is closely related to Vonk's multidimensional correlation function, $\gamma\left(r_{12}, r_{3}\right)$ ([59] and [9], p. 302). Similar to processing known from tomography, one could think of synthesizing the CDF from a complete set of IDFs according to Ruland [15], but a viable algorithm for this path has not yet been found.

Comparing the IDF to the CDF, the $1 \mathrm{D}$ derivative $d / d r_{3}$ of the electron density $\rho\left(r_{3}\right)$ is replaced by the gradient $\nabla \rho(\mathbf{r})$, as is the second derivative $d^{2} / d x^{2}$ in the 




Fig. 2.8 A particle-ghost autocorrelation of gradient vectors is generating the CDF. These vectors are emanating in normal direction from the surfaces of the particle and its displaced ghost. The ghost is displaced by the vector $\mathbf{r}$. The scalar product of the gradient vectors is vanishing everywhere, except for the regions with surface contact between particle and ghost

IDF by the Laplacian $\Delta$ in the CDF [13]. In analogy to the particle-ghost construction of the correlation function the construction of the CDF is readily demonstrated (Fig. 2.8). In a multiphase material the gradient field $\nabla \rho(\mathbf{r})$ is vanishing almost everywhere. Exceptions are the domain surfaces. They are continuously populated with gradient vectors, the lengths of which are proportional to the heights of the density jumps. Thus, the autocorrelation among the edge-enhanced structure and its ghost as a function of ghost displacement, $\mathbf{r}$, is approximately proportional to the area of surface contact and the product of the density jumps at the contact surface. Its sign is indicating if there is inner or outer contact. By definition of the CDF, outer contact is carrying the positive sign.

\subsubsection{Relation Between a CDF and IDFs}

Every radial, 1D slice through the center of a CDF

$$
\lceil z\rceil_{1}\left(r_{\psi, \varphi}\right)=g_{1}\left(r_{\psi, \varphi}\right)
$$

is an IDF, by definition. In the above equation, the slicing direction is indicated by a polar and an azimuthal angle, $\psi$ and $\varphi$, respectively. Of particular practical interest for the study of fibers is the cut of the CDF along the fiber axis,

$$
\lceil z\rceil_{1}\left(r_{3}\right)=z\left(0, r_{3}\right)=g_{1}\left(r_{3}\right)
$$

which describes the longitudinal structure of the material (cf. Sect. 2.6.2.1). In analogous manner the transverse structure of the fiber is described by the slice

$$
\lceil z\rceil_{2}\left(r_{12}\right)=z\left(0, r_{12}\right)=g_{2}\left(r_{12}\right)
$$



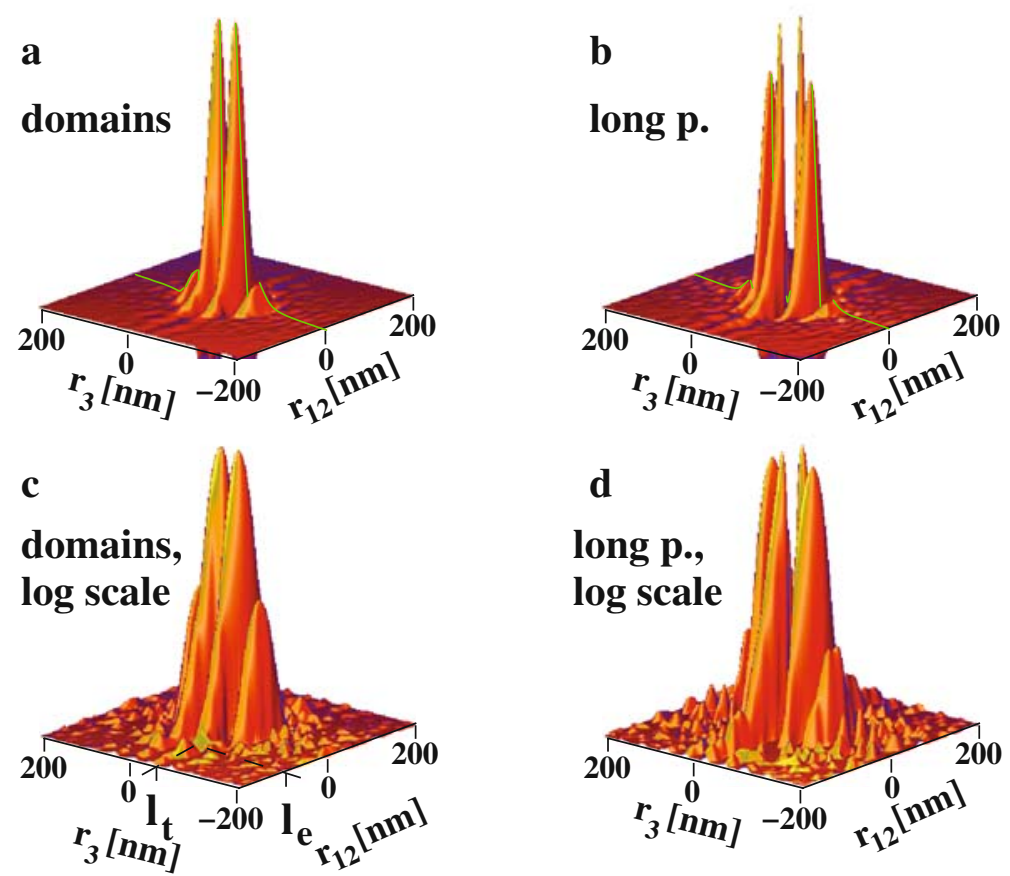

Fig. 2.9 Demonstration of a CDF. Data recorded during non-isothermal-oriented crystallization of polyethylene at $117^{\circ} \mathrm{C}$. Surface plots show the same CDF: (a) Linear scale viewed from the top. (b) Linear scale viewed from the bottom. (c) Viewed from the top, logarithmic scale. Indicated are the determination of the most probable layer thickness, $l_{t}$, and the maximum layer extension, $l_{e}$. (d) Viewed from the bottom, logarithmic scale. The IDF in fiber direction is indicated by a light line in (a) and (b) (Source: [60])

of the CDF. A typical CDF of a highly oriented semicrystalline polymer material is shown in Fig. 2.9. Viewed from the top the domain peaks are visible, whereas viewing a $\mathrm{CDF}$ from the bottom shows the long periods peaking out.

\subsubsection{Example of a CDF Analysis}

The Material of the Example

Poly(ether ester) (PEE) materials are thermoplastic elastomers. Fibers made from this class of multiblock copolymers are commercially available as Sympatex ${ }^{\circledR}$. Axle sleeves for automotive applications or gaskets are traded as Arnitel ${ }^{\circledR}$ or Hytrel ${ }^{\circledR}$. Polyether blocks form the soft phase (matrix). The polyester forms the hard domains which provide physical cross-linking of the chains. This nanostructure is the reason for the rubbery nature of the material. 

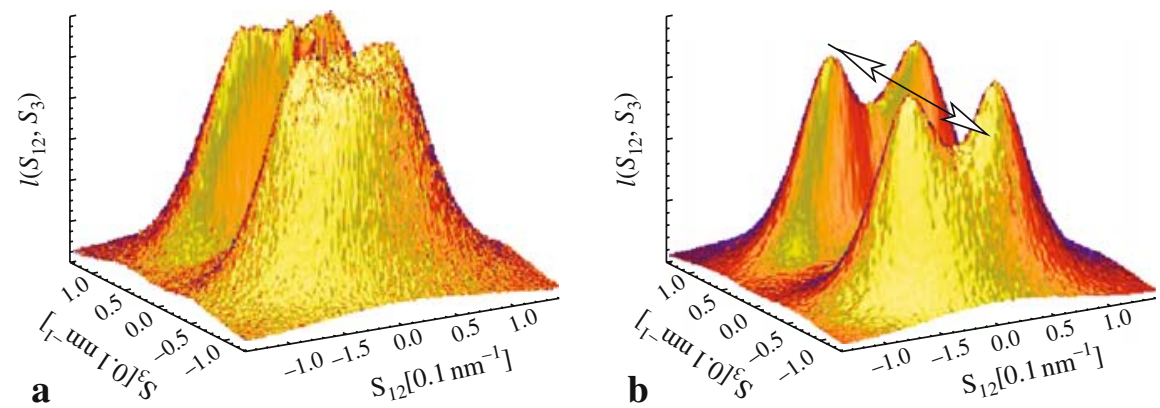

Fig. 2.10 Fiber scattering of PEE 1000/43: (a) at an elongation $\varepsilon=0.88$; (b) during relaxation from $\varepsilon=0.88$. The fiber direction is indicated by a double arrow. Visualized region: $-0.15 \mathrm{~nm}^{-1} \leq$ $s_{12}, s_{3} \leq 0.15 \mathrm{~nm}^{-1} . \varepsilon=\left(l-l_{0}\right) / l_{0}$, with $l_{0}$ and $l$ defined by the initial and the actual distance, respectively, between two fiducial marks on the sample

Figure 2.10 shows central sections of two original SAXS patterns of PEE $1000 / 43^{21}$ in strained and relaxed state. In the strained state (Fig. 2.10a) a "6-point diagram" is detected. During relaxation (Fig. 2.10b) a well-separated "4-point diagram" is observed. Interpretation of the patterns is restricted to description and speculation.

In an original paper [37] the longitudinal structure has been studied quantitatively as a function of elongation. In a follow-up study [14] the 3D CDF has been computed and analyzed. Figure 2.11 shows the 3D CDF with fiber symmetry computed from the scattering pattern in Fig. 2.10. The straining direction $r_{3}$ is indicated by the long arrow in the base plane. The observer is facing the domain peaks. Close to the

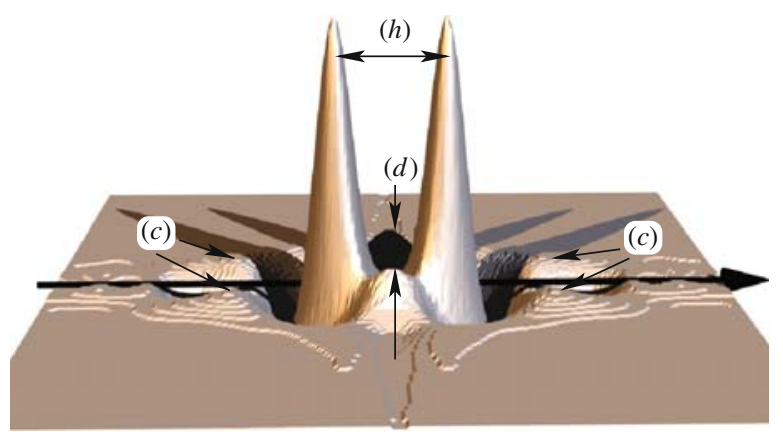

Fig. 2.11 PEE $1000 / 43$ at $\varepsilon=0.88$. CDF $z(\mathbf{r})$. The domain peaks are pointing upward: $(h)$ cylinder-height peaks; $(d)$ cylinder-diameter peaks; $(c)$ inter-domain correlation peaks. Displayed region: $\left|r_{12}, r_{3}\right| \leq 40 \mathrm{~nm}$

${ }^{21}$ PEEs are commonly characterized by two numbers (e.g., 1500/50). The first number reports the minimum quantization of the polyether blocks (meaning "the polyether blocks are multiples of $1500 \mathrm{~g} / \mathrm{mol}$ "), the second number indicates the mass fraction of the polyester hard phase (e.g., $50 \mathrm{wt} \%$ of polyester). 
origin the strong peaks on the meridian $(h)$ mark the correlation between opposite faces of the basic domains. Two equatorial peaks $(d)$ indicate the diameter of the domains. Because the height-to-diameter ratio is greater than 1, the basic domains are cylinders. Four correlation peaks $(c)$ are observed in an oblique angle with respect to the fiber axis. They indicate arrangement of domains. Their position shows that the closest neighbors of a cylinder are not found in straining direction, which would be indicative of a microfibrillar arrangement. Instead, the cylinders form a cluster with 3D short-range correlation. Such structural entities have been called a macrolattice by Wilke [61, 62]. The discussed peaks carry positive sign, because they describe chords that reach from the front face of a cylinder to the back face of a neighboring domain. The corresponding long periods show up as indentations observed at a shorter distance from the center, as they are measured "from front to front" of the domains. They are more easily observed after the CDF has been turned upside down (Fig. 2.12). Obviously the long periods in fiber direction (a) are less pronounced than the long periods in oblique direction (b). Moreover, the CDF shows that the topology does not contain long-ranging correlations among domains. In fiber direction there is a long period of $25 \mathrm{~nm}$ (a), but already the size of the domain behind it can no longer be determined. On the other hand, the arrangement of domains in oblique direction (b) shows better correlation: here not only the long period, but also the size of the cylinder behind it can be determined (Fig. 2.11(c)).

During the beamtime another scattering pattern has been exposed after unloading the material. The respective CDF is shown in Fig. 2.13. Compared with the data from the strained state, the positions of the oblique long periods do not move (b). This finding indicates that the central cylinders are surrounded by domains which are rigidly coupled to them. In the scattering pattern such a structural entity is not easily discriminated from the 4-point diagram of a stack of inclined lamellae. In this respect the $\mathrm{CDF}$ is much clearer.

Finally, we can compare the nanostructure in fiber direction after unloading with the nanostructure observed under mechanical load. The most striking variation is related to the strong long period (a), which is relaxing to half the value found in the elongated state. In addition to the strong long period, only in the unloaded

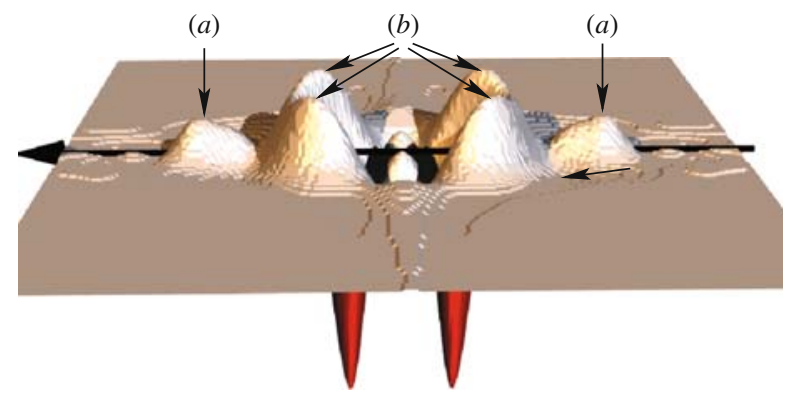

Fig. 2.12 CDF $-z(\mathbf{r})$ of PEE $1000 / 43$ at $\varepsilon=0.88$. The long-period peaks are pointing upward: (a) long period to the next neighbor in straining direction; (b) stronger long period to the closest neighbor (in oblique direction) 


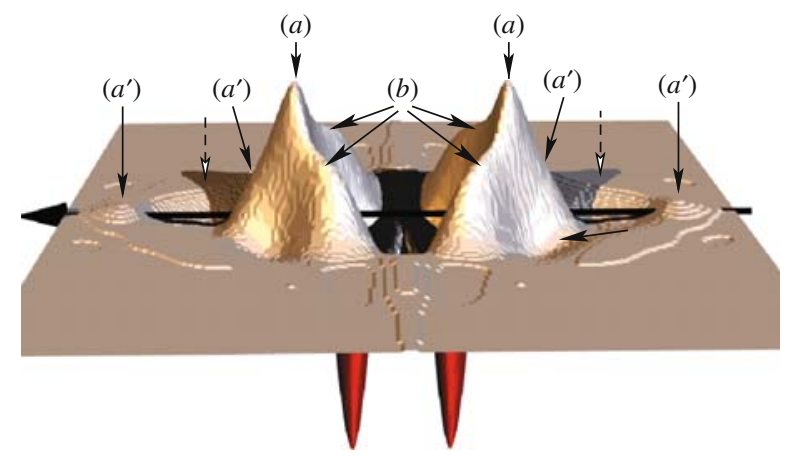

Fig. 2.13 $-z(\mathbf{r})$ for PEE 1000/43 recorded during relaxation of the material from a first elongation to $\varepsilon=0.88$. (a) Strongest long period in straining direction $(13 \mathrm{~nm})$ (dashed arrows with white head indicate the old positions of these peaks under strain); $\left(\mathrm{a}^{\prime}\right)$ : the best-correlated long period in fiber direction $(17 \mathrm{~nm})$, because it shows a second order; (b) oblique long period that is immovable in the straining experiment

material another long period is found $\left(a^{\prime}\right)$, for which even the second order is visible. Thus, the corresponding structural entities are built from domains with already a considerable range of correlation which are arranged along the straining direction. This is the topological definition of a microfibril [63]. As the material becomes strained, the softer matter between the domains is elongated by different amounts and the longitudinal correlation gets lost. Thus, the semi-quantitative analysis of the CDF returns a detailed view on the nanostructure evolution under load. More examples of the CDF method can be found in a growing number of original studies [39-46, 60, 64-66].

\section{References}

1. Debye P., Menke H. Erg. Techn. Röntgenkunde 2, 1-22 (1931). 23, 32

2. Hammersley A.P. FIT2D V12.012 Reference Manual, http://www.esrf.fr/computing/scientific/ FIT2D/. 27

3. Alexander L.E. X-Ray Diffraction Methods in Polymer Science. Wiley, New York (1979). 28, 29

4. Fraser R.D., Macrae T.P., Miller A., Rowlands R.J., J. Appl. Cryst. 9, 81-94 (1976). 28

5. Buhmann M.D. Acta Numerica 9, 1-38 (2000). 28

6. Hosemann R., Bagchi S.N. Direct Analysis of Diffraction by Matter. North-Holland, Amsterdam (1962). 29, 41

7. Guinier A. X-Ray Diffraction. Freeman, San Francisco (1963). 29

8. Feigin L.A., Svergun D.I. Structure Analysis by Small-Angle X-Ray and Neutron Scattering. Plenum Press, New York (1987). 29

9. Baltá Calleja F.J., Vonk C.G. X-Ray Scattering of Synthetic Polymers. Elsevier, Amsterdam (1989). 29, 34, 41, 44, 53

10. Stribeck N. X-Ray Scattering of Soft Matter. Springer, Heidelberg, New York (2007). 29, 32, 41, 44, 45, 47, 5

11. Debye P., Bueche A.M. J. Appl. Phys. 20, 518-525 (1949). 31

12. Porod G. Colloid Polym. Sci. 124, 83-114 (1951). 31 
13. Stribeck N. J. Appl. Cryst. 34, 496-503 (2001). 31, 38, 52, 54

14. Stribeck N., Fakirov S. Macromolecules 34, 7758-7761 (2001). 31, 56

15. Ruland W. Colloid Polym. Sci. 255, 417-427 (1977). 31, 46, 53

16. Ruland W. Colloid Polym. Sci. 256, 932-936 (1978). 31, 53

17. Stribeck N., Ruland W. J. Appl. Cryst. 11, 535-539 (1978). 31

18. Méring J., Tchoubar-Vallat D. C. R. Acad. Sc. Paris 261, 3096-3099 (1965). 31, 53

19. Méring J., Tchoubar-Vallat D. C. R. Acad. Sc. Paris 262, 1703-1706 (1966). 31

20. Méring J., Tchoubar D. J. Appl. Cryst. 1, 153-165 (1968). 31, 53

21. Tchoubar D., Méring J. J. Appl. Cryst. 2, 128-138 (1969). 31, 48, 53

22. Bonart R. Kolloid Z. u. Z. Polymere 211, 14-33 (1966). 33, 34, 37, 41, 43, 44, 47

23. Conner W.C., Webb S.W., Spanne P., Jones K.W. Macromolecules 23, $4742-4747$ (1990). 34

24. Schroer C.G., Kuhlmann M., Roth S.V., Gehrke R., Stribeck N., Almendarez Camarillo A., Lengeler B. Appl. Phys. Lett. 88, 164102 (2006). 34

25. Spontak R.J., Williams M.C., Agard D.A. Polymer 29, 387-395 (1988). 34

26. Stokes A.R. Proc. Phys. Soc. 61, 382-391 (1948). 39

27. Deng Y., He G., Kuppusamy P., Zweier J.L. Magn. Reson. Med. 50, 444-448 (2003). 40

28. VNI. PV-WAVE Manuals. V 7.5, Boulder, Colorado (2007). 40, 41, 42, 43

29. RSI. Interactive Data Language IDL. V 6.1, Boulder, Colorado (2004). 40

30. Burger H.C., van Cittert P.H. Z. Phys. 79, 722 (1932). 40

31. Ergun S. J. Appl. Cryst. 1, 19-23 (1968). 40

32. Glatter O. J. Appl. Cryst. 14, 101-108 (1981). 40, 41

33. Guinier A., Fournet G. Small-Angle Scattering of X-Rays. Chapman and Hall, London (1955). 41

34. Glatter O., Kratky O. (Eds.), Small Angle X-ray Scattering. Academic Press, London (1982). 41, 44, 47

35. Stribeck N. Downloads, http://www.chemie.uni-hamburg.de/tmc/stribeck/dl (2008). 44

36. Stribeck N. Colloid Polym. Sci. 271, 1007-1023 (1993). 46, 48

37. Stribeck N., Fakirov S., Sapoundjieva D. Macromolecules 32, 3368-3378 (1999). 46, 56

38. Verma R., Marand H., Hsiao B. Macromolecules 29, 7767-7775 (1996). 46

39. Stribeck N., Buzdugan E., Ghioca P., Serban S., Gehrke R. Macromol. Chem. Phys. 203, 636-644 (2002). 46, 58

40. Stribeck N., Bayer R., von Krosigk G., Gehrke R. Polymer 43, 3779-3784 (2002). 46, 58

41. Barbi V., Funari S.S., Gehrke R., Scharnagl N., Stribeck N. Polymer 44, 4853-4861 (2003). 46, 58

42. Stribeck N., Androsch R., Funari S.S. Macromol. Chem. Phys. 204, 1202-1216 (2003). 46, 58

43. Stribeck N., Funari S.S. J. Polym. Sci. Part B: Polym. Phys. 41, 1947-1954 (2003). 46, 58

44. Stribeck N. Macromol. Chem. Phys. 205, 1455-1462 (2004). 46, 58

45. Stribeck N., Almendarez Camarillo A., Bayer R. Macromol. Chem. Phys. 205, 1463-1470 (2004). 46, 58

46. Stribeck N., Bösecke P., Bayer R., Almendarez Camarillo A. Progr. Coll. Polym. Sci. 130, 127-139 (2005). 46, 58

47. Almendarez Camarillo A., Roth S.V., Bösecke P., Buchner S., Krenn K., Gehrke R., Stribeck N. J. Mater. Sci. 42, 6212-6221 (2007). 46

48. Stribeck N. J. Polym. Sci., Part B: Polym. Phys. 37, 975-981 (1999). 47, 48, 50

49. Wu J., Schultz J.M., Yeh F., Hsiao B.S., Chu B. Macromolecules 33, 1765-1777 (2000). 47

50. Kratky O., Porod G., Kahovec L. Z. Elektrochemie 55, 53-59 (1951). 47

51. Ruland W. J. Appl. Cryst. 4, 70-73 (1971). 47, 52

52. Stribeck N., Reimers C., Ghioca P., Buzdugan E. J. Polym. Sci. B: Polym. Phys. 36, 1423 1432 (1998). 47

53. Schmidt P.W. J. Math. Phys. 8, 475-477 (1967). 48

54. Marichev O. I. Handbook of Integral Transforms of Higher Transcendental Functions. Ellis Horwood Ltd., Chichester (1983). 48

55. Glatter O. J. Appl. Cryst. 7, 147-153 (1974). 48

56. Apostolov A.A., Boneva D., Baltá Calleja F.J., Krumova M., Fakirov S. J. Macromol. Sci. Phys. 37, 543-555 (1998). 50

57. Koberstein J.T., Morra B., Stein R.S. J. Appl. Cryst. 13, 34-45 (1980). 52 
58. Mering J., Tchoubar D., Schiller C. Bull. Soc. Fr. Mineral. Cristallogr. XC, 436-444 (1967). 53

59. Vonk C.G. Colloid Polym. Sci. 257, 1021-1032 (1979). 53

60. Stribeck N., Almendarez Camarillo A., Cunis S., Bayer R.K., Gehrke R. Macromol. Chem. Phys. 205, 1445-1454 (2004). 55, 58

61. Fronk W., Wilke W. Colloid Polym. Sci. 263, 97-108 (1985). 57

62. Wilke W., Bratrich M. J. Appl. Cryst. 24, 645-650 (1991). 57

63. Peterlin A. Text. Res. J. 42, 20-30 (1972). 58

64. Barbi V., Funari S.S., Gehrke R., Scharnagl N., Stribeck N. Macromolecules 38, 749-758 (2003). 58

65. Stribeck N., Fakirov S., Apostolov A.A., Denchev Z., Gehrke R. Macromol. Chem. Phys. 204, 1000-1013 (2003). 58

66. Stribeck N., Bayer R., Bösecke P., Almendarez Camarillo A. Polymer 46, 2579-2583 (2005). 58 